TRANSACTIONS OF THE

AMERICAN MATHEMATICAL SOCIETY

Volume 365, Number 10, October 2013, Pages 5277-5312

S 0002-9947(2013)05810-2

Article electronically published on April 1, 2013

\title{
NON-AUTONOMOUS MORSE-DECOMPOSITION AND LYAPUNOV FUNCTIONS FOR GRADIENT-LIKE PROCESSES
}

\author{
E. R. ARAGÃO-COSTA, T. CARABAllo, A. N. CARVAlHO, AND J. A. LANGA
}

\begin{abstract}
We define (time dependent) Morse-decompositions for non-autonomous evolution processes (non-autonomous dynamical systems) and prove that a non-autonomous gradient-like evolution process possesses a Morsedecomposition on the associated pullback attractor. We also prove the existence of an associated Lyapunov function which describes the gradient behavior of the system. Finally, we apply these abstract results to non-autonomous perturbations of autonomous gradient-like evolution processes (semigroups or autonomous dynamical systems).
\end{abstract}

\section{INTRODUCTION}

The Fundamental Theorem of Dynamical Systems (see 8]) describes the flow of autonomous reversible dynamical systems (groups) in compact metric spaces as a decomposition of an ordered family of isolated invariant sets and connections between them which respects their ordering. In the terminology of [8], this is called a Morse-decomposition of a compact invariant set. The extension of this result to general autonomous dynamical systems (or semigroups) is done in [1 (see also 20] for the Morse-decomposition for semigroups in compact metric spaces). For autonomous dynamical systems, the Morse-decomposition plays a fundamental role in the process of understanding the structure of invariant sets. It allows us to decompose the dynamics into a gradient part and the dynamics in smaller isolated invariant sets, reducing the study of the structure of the attractors to the study of the structure of these isolated invariant sets.

The aim of this paper is to extend the notion of Morse-decomposition to nonautonomous dynamical systems (or non-autonomous evolution processes) and to show the pullback attractors for some of these non-autonomous dynamical systems

Received by the editors April 8, 2011 and, in revised form, September 21, 2011 and February 3,2012 .

2010 Mathematics Subject Classification. Primary 37B25, 37B35; Secondary 37B55, 35B40, $35 \mathrm{~B} 41$.

Key words and phrases. Non-autonomous Morse decomposition, Lyapunov functions for nonautonomous dynamical systems, robustness of dynamical structures.

The first author was partially supported by CAPES/DGU 267/2008 and FAPESP 2008/502480, Brazil.

The second author was partially supported by FEDER and Ministerio de Ciencia e Innovación grant \# MTM2008-0088, \# MTM2011-22411, PBH2006-0003-PC, and Junta de Andalucía grants \# P07-FQM-02468, \# FQM314 and HF2008-0039, Spain.

The third author was partially supported by CNPq 302022/2008-2, CAPES/DGU 267/2008 and FAPESP 2008/55516-3, Brazil and Junta de Andalucía grant \# P07-FQM-02468.

The fourth author was partially supported by FEDER and Ministerio de Ciencia e Innovación grants \# MTM2008-0088, \# MTM2011-22411, \# PBH2006-0003-PC, and Junta de Andalucía grants \# P07-FQM-02468, \# FQM314 and HF2008-0039, Spain.

(C) 2013 American Mathematical Society Reverts to public domain 28 years from publication 
can also be decomposed as an ordered family of isolated invariant families and connections between them respecting their ordering. In particular, under some mild additional assumptions, we construct a non-autonomous Lyapunov function for the non-autonomous evolution process with a Morse-decomposition. We show that this decomposition is observed for non-autonomous perturbations of gradient autonomous dynamical systems (or gradient semigroups). Some practical examples are also presented.

Our results contrast with those of 19 because, in our case, the definition of local attractors takes into account only the usual notion of attraction instead of using past and forwards notions. We also do not impose the reversibility required in [19. Our results can be applied (under mild assumptions) to perturbations of autonomous dynamical systems (which cannot be said for the results in [19]). Furthermore, in the case of perturbations of autonomous dynamical systems and under mild assumptions, we prove some sort of continuity of the Morse-decomposition.

To better describe the results in the paper we will need to introduce some terminology and a few definitions. Let $X$ be a metric space with metric $\mathbf{d}: X \times X \rightarrow \mathbb{R}^{+}$, where $\mathbb{R}^{+}:=[0, \infty)$, and denote by $\mathcal{C}(X)$ the set of continuous maps from $X$ into $X$. Given a subset $A \subset X$, the $\epsilon$-neighborhood of $A$ is the set $\mathcal{O}_{\epsilon}(A):=\{z \in X$ : $\mathbf{d}(z, a)<\epsilon$ for some $a \in A\}$.

An evolution process in a metric space $X$ is a two-parameter family $\{T(t, s)$ : $t \geq s\}$ in $\mathcal{C}(X)$ such that

1) $T(t, t)=I$, for all $t \in \mathbb{R}$,

2) $T(t, \sigma) T(\sigma, s)=T(t, s)$, for $t \geq \sigma \geq s$, and

3) $\mathcal{P} \times X \ni((t, s), x) \mapsto T(t, s) x \in X$ is continuous, where $\mathcal{P}:=\left\{(t, s) \in \mathbb{R}^{2}: t \geq s\right\}$.

An evolution process $\{T(t, s): t \geq s\}$ is autonomous when $T(t, s)=T(t-s, 0)$ for all $t \geq s$, otherwise it is non-autonomous. A semigroup is a family $\{S(t): t \geq 0\} \subset$ $\mathcal{C}(X)$ such that

1) $S(0)=I$, for all $t \in \mathbb{R}$,

2) $S(t+s)=S(t) S(s), t, s \geq 0$, and

3) $\mathbb{R}^{+} \times X \ni(t, x) \mapsto S(t) x \in X$ is continuous.

Note that $\{T(t, s): t \geq s\}$ is an autonomous evolution process iff $\{T(t, 0): t \geq 0\}$ is a semigroup. A continuous function $\xi: \mathbb{R} \rightarrow X$ is a global solution for the evolution process $\{T(t, s): t \geq s\}$ when it satisfies

$$
T(t, s) \xi(s)=\xi(t), \text { for all }(t, s) \in \mathcal{P} .
$$

Next we recall the definition of Hausdorff semidistance and Hausdorff distance. Given $A, B \subset X$, the Hausdorff semidistance from $A$ to $B$ is given by

$$
\operatorname{dist}(A, B):=\sup _{a \in A} \inf _{b \in B} \mathbf{d}(a, b)=\sup _{a \in A} \mathbf{d}(a, B),
$$

where $\mathbf{d}(a, B):=\inf _{b \in B} \mathbf{d}(a, b)$ is the usual distance from the point $a$ to the set $B$, and the Hausdorff distance between $A$ and $B$ is defined by

$$
\mathbf{d}_{\mathrm{H}}(A, B):=\operatorname{dist}(A, B)+\operatorname{dist}(B, A) .
$$

Definition 1.1. A family $\Xi:=\{\Xi(t): t \in \mathbb{R}\}$ of subsets of $X$ is an invariant family for the evolution process $\{T(t, s): t \geq s\}$ when $T(t, s) \Xi(s)=\Xi(t)$ for all $t \geq s$. An 
invariant family $\{\mathscr{A}(t): t \in \mathbb{R}\}$ is a pullback attractor when, for each $t \in \mathbb{R}, \mathscr{A}(t)$ is compact, pullback attracts bounded subsets of $X$; that is,

$\lim _{s \rightarrow-\infty} \operatorname{dist}(T(t, s) B, \mathscr{A}(t))=0$, for each $t \in \mathbb{R}$ and bounded subset $B$ of $X$,

and $\{\mathscr{A}(t): t \in \mathbb{R}\}$ is minimal among all closed invariant families $\{\mathscr{C}(t): t \in \mathbb{R}\}$ with the property that $\mathscr{C}(t)$ pullback attracts bounded subsets of $X$ for each $t \in \mathbb{R}$.

Recall that, if $\{S(t): t \geq 0\}$ is a semigroup in a metric space $X$, it is said that $A \subset X$ is invariant for $\{S(t): t \geq 0\}$ if $S(t) A=A$, for all $t \geq 0$. It is important to notice that, in the autonomous context, the non-autonomous notion of invariance also allows for non-constant families.

The notion of an unstable set of an invariant family plays a key role in the definition of attractor-repeller pairs. On their turn, the attractor-repeller pairs enter in an essential way in the definition of Morse-decomposition for the pullback attractor of a non-autonomous evolution process. Next we define these concepts beginning with the definition of an unstable set of an invariant family.

Definition 1.2. Let $\{T(t, s): t \geq s\}$ be an evolution process. The unstable set of an invariant family $\Xi=\{\Xi(t): t \in \mathbb{R}\}$ is the set

$$
\begin{gathered}
W^{\mathrm{u}}(\Xi):=\{(t, z) \in \mathbb{R} \times X: \text { there is a global solution } \xi: \mathbb{R} \rightarrow X \\
\text { such that } \left.\xi(t)=z \text { and } \lim _{s \rightarrow-\infty} \operatorname{dist}(\xi(s), \Xi(s))=0\right\} .
\end{gathered}
$$

Also, $W^{\mathrm{u}}(\Xi)(t):=\left\{z \in X:(t, z) \in W^{\mathrm{u}}(\Xi)\right\}$ for each $t \in \mathbb{R}$.

Now we introduce the notions of isolated invariant sets and disjoint families of isolated invariant sets.

Definition 1.3. Let $\Xi:=\{\Xi(t): t \in \mathbb{R}\}$ be an invariant family for the evolution process $\{T(t, s): t \geq s\} . \Xi$ is an isolated invariant family if there exists a $\delta>0$ with the property that any global solution $\xi: \mathbb{R} \rightarrow X$ with $\xi(t) \in \mathcal{O}_{\delta}(\Xi(t))$ must satisfy that $\xi(t) \in \Xi(t)$ for all $t \in \mathbb{R}$. A set $\Xi=\left\{\Xi_{1}, \cdots, \Xi_{n}\right\}$ is said to be a disjoint set of isolated invariant families if each $\Xi_{i}, 1 \leq i \leq n$, is an isolated invariant family and there exists $\delta>0$ such that $\mathcal{O}_{\delta}\left(\Xi_{i}(t)\right) \cap \mathcal{O}_{\delta}\left(\Xi_{j}(t)\right)=\varnothing$, for all $t \in \mathbb{R}$ and for $1 \leq i<j \leq n$.

Remark 1.4. Let $\{S(t): t \geq 0\}$ be a semigroup in a metric space $X$. It is important to notice that:

(1) A subset $A$ of $X$ is an isolated invariant set for $\{S(t): t \geq 0\}$ if it is invariant and there is a $\delta>0$ such that $A$ is the maximal invariant set for $\{S(t): t \geq 0\}$ in $\mathcal{O}_{\delta}(A)$.

(2) If $\xi: \mathbb{R} \rightarrow X$ is a uniformly continuous global solution for $\{S(t): t \geq 0\}$, then $\{\{\xi(t)\}: t \in \mathbb{R}\}, t \in \mathbb{R}$, is an invariant family which is not isolated. Any isolated invariant family $\{\Xi(t): t \in \mathbb{R}\}$ such that $\xi(t) \in \Xi(t)$ for all $t \in \mathbb{R}$ must satisfy that $\xi(\mathbb{R}) \subset \Xi(t)$ for all $t \in \mathbb{R}$. In the autonomous context this remark says that, in some sense, the two notions of isolated invariant sets (autonomous and non-autonomous) are similar.

(3) It is easy to see that if $A \subset X$ and $\delta>0$ is such that $A$ is the maximal invariant set for $\{S(t): t \geq 0\}$ in $\mathcal{O}_{\delta}(A)$, then

- If $\delta^{\prime}<\delta$ and $x \in X$ is such that $\gamma^{+}(x) \subset \mathcal{O}_{\delta^{\prime}}(A)$, then $\mathbf{d}(T(t) x, A) \stackrel{t \rightarrow \infty}{\longrightarrow} 0$.

- If $\delta^{\prime}<\delta$ and there is a global solution $\xi: \mathbb{R} \rightarrow X$ such that $\xi\left(\mathbb{R}^{-}\right) \subset$ $\mathcal{O}_{\delta^{\prime}}(A)$, then $\mathbf{d}(\xi(t), A) \stackrel{t \rightarrow-\infty}{\longrightarrow} 0$. 
(4) The preceding remark ensures that a center in a two dimensional dynamical system or an equilibrium which is part of a continuum of equilibria will not be an isolated invariant set.

The notions of a local attractor and of an atractor-repeller pair are defined next.

Definition 1.5. Let $\{T(t, s): t \geq s\}$ be an evolution process in a metric space $X$ with a pullback attractor $\{\mathscr{A}(t): t \in \mathbb{R}\}$. We say that an isolated invariant family $A:=\{A(t): t \in \mathbb{R}\}$, with $A(t) \subset \mathscr{A}(t)$ for all $t \in \mathbb{R}$, is a (non-autonomous) local attractor if $W^{u}(A)(t)=A(t)$ for all $t \in \mathbb{R}$. by

If $A$ is a local attractor, we define its associated repeller $A^{*}:=\left\{A^{*}(t): t \in \mathbb{R}\right\}$

$A^{*}(t):=\{z \in \mathscr{A}(t): \operatorname{dist}(T(r+t, t) z, A(r+t)) \nrightarrow 0$ as $r \rightarrow \infty\}$, for each $t \in \mathbb{R}$.

The pair $\left(A, A^{*}\right)$ is called an attractor-repeller pair.

The following result ensures that the above definition extends its autonomous counterpart. We sketch its proof for completeness (see [1, Lemma 2.15] for a complete proof).

Theorem 1.6. Let $\{S(t): t \geq 0\}$ be a semigroup in a metric space $X$ with a global attractor $\mathscr{A}$. An isolated invariant set $A \subset \mathscr{A}$ is a local attractor (autonomous) iff the constant family $\{A(t): A(t)=A, t \in \mathbb{R}\}$ is a local attractor for $\{T(t, s)$ : $T(t, s):=S(t-s)$ for $t \geq s\}$, in the sense of Definition 1.5 .

Proof. Note that

$$
W^{u}(A)=W^{u}(\{A(t): t \in \mathbb{R}\})(t), \quad \forall t \in \mathbb{R},
$$

where $W^{u}(A):=\{z \in A$ : there is a global solution $\xi: \mathbb{R} \rightarrow X$ with $\xi(0)=z$ and $\left.\lim _{t \rightarrow-\infty} d(\xi(t), A)=0\right\}$ and that if $A$ is an isolated invariant set for $S(\cdot)$ such that $W^{u}(A)=A$, then for every $\delta>0$ there exists $\delta^{\prime} \in(0, \delta)$ with

$$
\gamma^{+}\left(\mathcal{O}_{\delta^{\prime}}(A)\right) \subset \mathcal{O}_{\delta}(A) .
$$

The proof now follows easily from the fact that $\omega\left(\mathcal{O}_{\delta^{\prime}}(A)\right)$ attracts $\mathcal{O}_{\delta^{\prime}}(A)$ and is invariant (hence it must be contained in $A$ ).

For autonomous evolution processes (semigroups) a repeller is automatically closed, but that is not the case for non-autonomous evolution processes. However, if there exists $\varepsilon>0$ such that $\mathcal{O}_{\varepsilon}(A(t)) \cap \mathcal{O}_{\varepsilon}\left(A^{*}(t)\right)=\varnothing$, for all $t \in \mathbb{R}$, then $A^{*}(t)$ is closed for each $t \in \mathbb{R}$.

Now, we define the notion of Morse-decomposition for non-autonomous evolution processes.

Definition 1.7. Let $\{T(t, s): t \geq s\}$ be an evolution process in $X$ with a pullback attractor $\{\mathscr{A}(t): t \in \mathbb{R}\}$ and let $A_{0}=\left\{A_{0}(t): t \in \mathbb{R}\right\}, A_{1}=\left\{A_{1}(t): t \in \mathbb{R}\right\}, \cdots$, $A_{n}=\left\{A_{n}(t): t \in \mathbb{R}\right\}$ be $n+1$ local attractors with $\varnothing=A_{0}(t) \subset A_{1}(t) \subset \cdots \subset$ $A_{n}(t)=\mathscr{A}(t)$ for each $t \in \mathbb{R}$.

Define $\Xi_{j}(t):=A_{j}(t) \cap A_{j-1}^{*}(t)$ for each $t \in \mathbb{R}$ and $j=1,2, \cdots, n$. The ordered set of invariant families $\Xi:=\left\{\Xi_{1}, \Xi_{2}, \cdots, \Xi_{n}\right\}$ is called a Morse-decomposition for the pullback attractor $\{\mathscr{A}(t): t \in \mathbb{R}\}$. 
In Section 2 we give sufficient conditions to obtain a Morse-decomposition for the pullback attractor of a gradient-like evolution process.

Section 3 is devoted to obtain, under some mild assumptions, a Lyapunov function for a gradient-like (non-autonomous) evolution process restricted to the pullback attractor. We also give conditions so that the Lyapunov function is defined and is continuous in $\mathbb{R} \times X$.

We prove in Section 4 that the results of Section 2 can be applied (under natural assumptions) to non-autonomous perturbations of autonomous gradient-like evolution processes. In this situation, thanks to the results from Section 3 , the Lyapunov function can be defined and is continuous in $\mathbb{R} \times X$. The continuity of the Lyapunov function under perturbation is also considered.

Section 5 exhibits some concrete examples where the theory developed in the previous sections can be applied.

\section{Morse-DeCOMPosition of PULlbaCK ATtRACTORS FOR GENERALIZED GRADIENT-LIKE EVOLUTION PROCESSES}

We first introduce the notion of a gradient-like process (see [5]). Let $\{T(t, s): t \geq$ $s\}$ be an evolution process with a pullback attractor $\{\mathscr{A}(t): t \in \mathbb{R}\}$ which contains a disjoint set of isolated invariant families $\boldsymbol{\Xi}=\left\{\Xi_{1}, \cdots, \Xi_{n}\right\}$, that is, $\Xi_{i}(t) \subset \mathscr{A}(t)$ for each $i$ and $t$. We can now define the concept of pinned-chain recurrence (see [1, 5, 8, 15] for similar concepts in the autonomous case):

Definition 2.1. Let $\delta$ be as in Definition 1.3 and fix $\epsilon_{0} \in(0, \delta)$. For $\Xi \in \boldsymbol{\Xi}$ and $\epsilon \in\left(0, \epsilon_{0}\right)$, an $\epsilon$-pinned-chain from $\Xi$ to $\Xi$ is a sequence $\ell_{i}, 1 \leq i \leq k$, in $\{1, \cdots, n\}$, a sequence of real numbers $t_{i}, \sigma_{i}, \tau_{i}$ with $\tau_{i}<\sigma_{i}<t_{i}$, and a sequence of points $z_{i} \in X, 1 \leq i \leq k$, such that $z_{i} \in \mathcal{O}_{\epsilon}\left(\Xi_{\ell_{i}}\left(\tau_{i}\right)\right), T\left(\sigma_{i}, \tau_{i}\right) z_{i} \notin \mathcal{O}_{\epsilon_{0}}\left(\bigcup_{i=1}^{n} \Xi_{i}\left(\sigma_{i}\right)\right)$ and $T\left(t_{i}, \tau_{i}\right) z_{i} \in \mathcal{O}_{\epsilon}\left(\Xi_{\ell_{i+1}}\left(t_{i}\right)\right), 1 \leq i \leq k$, with $\Xi=\Xi_{\ell_{k+1}}=\Xi_{\ell_{1}}$. We say that $\Xi \in \boldsymbol{\Xi}$ is pinned-chain recurrent if there is an $\epsilon_{0} \in(0, \delta)$ and $\epsilon$-pinned-chain from $\Xi$ to $\Xi$ for each $\epsilon \in\left(0, \epsilon_{0}\right)$.

Remark 2.2. We note that the introduction of $\epsilon_{0}$ in the above definition is only needed to account for the case $k=1$. When $k>1$, it is automatically true that the solution must leave $\mathcal{O}_{\epsilon_{0}}\left(\bigcup_{i=1}^{n} \Xi_{i}(t)\right)$, for some $t \in \mathbb{R}$, while going from one isolated invariant family to another.

Before we proceed let us point out the distinctions between the notion of pinnedchain recurrence introduced in the above definition and the notion of chain recurrence defined in 8 . We start with the definition of chain recurrence given in 8$]$.

Definition 2.3 (Conley). Let $\{S(t): t \in \mathbb{R}\}$ be a group in a metric space $X$ and $\Xi \subset X$ be a compact invariant set. Given $\epsilon>0, t>0$ and $\xi, \xi^{\prime} \in \Xi$, an $(\epsilon, t)$-chain from $\xi$ to $\xi^{\prime}$ is a sequence $\xi=\xi_{0}, \xi_{1}, \cdots, \xi_{n}=\xi^{\prime} ; t_{1}, \cdots, t_{n}$ such that $t_{i} \geq t$ for all $i$ and $\mathbf{d}\left(S\left(t_{i}\right) \xi_{i-1}, \xi_{i}\right)<\epsilon, i=1, \cdots, n$. A subset $R(\Xi)$ of an invariant set $\Xi$ is called chain recurrent if for any $\xi \in R(\Xi), \epsilon>0$ and $t>0$ there is a $(\epsilon, t)$-chain from $\xi$ to itself.

Remark 2.4. The notions of pinned-chain recurrence and chain recurrence are different, even in the autonomous context. However, the first is strongly inspired by the second. In the pinned-chain recurrence notion the jumping points are pinned in the isolated invariant sets, and in chain recurrence notion the jumping points occur around points which are not pinned. 
Another important difference is that an equilibrium is always chain recurrent (Conley's concept), whereas they may not be pinned-chain recurrent (our concept). In fact, for gradient semigroups having a global attractor and with a finite set of equilibria, no equilibria is pinned-chain recurrent. To be pinned-chain recurrent there should exist solutions starting arbitrarily close to the isolated invariant set that leave a fixed neighborhood of it and come back arbitrarily close (possibly jumping only near other isolated invariant sets).

The notion of pinned-chain recurrence, in the autonomous case, is closely related to the existence of homoclinic structures, as shown in Lemma 2.2 in [5]. It is tailored to describe the dynamical properties of a gradient semigroup, without a priori requiring the existence of a Lyapunov function.

Definition 2.5. Let $X$ be a metric space and $\{T(t, s): t \geq s\}$ be an evolution process in $X$ with a pullback attractor $\{\mathscr{A}(t): t \in \mathbb{R}\}$ and a disjoint set of isolated invariant families $\boldsymbol{\Xi}=\left\{\Xi_{1}, \cdots, \Xi_{n}\right\}$ in $\{\mathscr{A}(t): t \in \mathbb{R}\}$. We say that $\{T(t, s): t \geq s\}$ is a gradient-like evolution process with respect to $\Xi$ if the following two conditions are satisfied:

(H1) Any global solution $\xi: \mathbb{R} \rightarrow X$ in $\{\mathscr{A}(t): t \in \mathbb{R}\}$ satisfies

$$
\lim _{t \rightarrow-\infty} \operatorname{dist}\left(\xi(t), \Xi_{i}(t)\right)=0 \text { and } \lim _{t \rightarrow \infty} \operatorname{dist}\left(\xi(t), \Xi_{j}(t)\right)=0,
$$

for some $1 \leq i, j \leq n$.

(H2) $\Xi=\left\{\Xi_{1}, \cdots, \Xi_{n}\right\}$ does not contain any pinned-chain recurrent isolated invariant family.

Let us now define the notion of a homoclinic structure.

Definition 2.6. Let $\{T(t, s): t \geq s\}$ be an evolution process which possesses a disjoint set of isolated invariant families $\Xi=\left\{\Xi_{1}, \cdots, \Xi_{n}\right\}$. A homoclinic structure associated to $\boldsymbol{\Xi}$ is a finite subset $\left\{\Xi_{i_{1}}, \cdots, \Xi_{i_{p}}\right\}$ of $\boldsymbol{\Xi}$ together with a set of global solutions $\left\{\xi_{1}, \cdots, \xi_{p}\right\}$ such that

$$
\lim _{t \rightarrow-\infty} \operatorname{dist}\left(\xi_{k}(t), \Xi_{i_{k}}(t)\right)=0 \text { and } \lim _{t \rightarrow \infty} \operatorname{dist}\left(\xi_{k}(t), \Xi_{i_{k+1}}(t)\right)=0,1 \leq k \leq p,
$$

where $\Xi_{i_{p+1}}:=\Xi_{i_{1}}$.

Next we prove the invariance of the repeller $A^{*}$ of a local attractor $A$.

Proposition 2.7. Let $\{T(t, s): t \geq s\}$ be an evolution process with a pullback attractor $\{\mathscr{A}(t): t \in \mathbb{R}\}$ and let $A=\{A(t): t \in \mathbb{R}\}$ be a local attractor. Then, the repeller $A^{*}$ of $A$ is invariant.

Proof. If $A^{*}\left(t_{0}\right)$ is empty for some $t_{0} \in \mathbb{R}$, then it is empty for all $t \in \mathbb{R}$ and the proof is obvious. Assume that $A^{*}(t)$ is non-empty for all $t \in \mathbb{R}$, and let $s \in \mathbb{R}, t \geq s$ and $w \in A^{*}(s)$. If $T(t, s) w \notin A^{*}(t)$ we have that

$$
\lim _{\tau \rightarrow \infty} \operatorname{dist}(T(\tau, t) T(t, s) w, A(\tau))=0,
$$

that is,

$$
\lim _{\tau \rightarrow \infty} \operatorname{dist}(T(\tau, s) w, A(\tau))=0,
$$

which contradicts the fact that $w \in A^{*}(s)$ and proves that $T(t, s) A^{*}(s) \subset A^{*}(t)$. 
Conversely, if $z \in A^{*}(t) \subset \mathscr{A}(t)=T(t, s) \mathscr{A}(s)$, let $w \in \mathscr{A}(s)$ be such that $z=T(t, s) w$. It follows that $w \in A^{*}(s)$, for otherwise

$$
\begin{aligned}
0 & =\lim _{\tau \rightarrow \infty} \operatorname{dist}(T(\tau, s) w, A(\tau)) \\
& =\lim _{\tau \rightarrow \infty} \operatorname{dist}(T(\tau, t) T(t, s) w, A(\tau))=\lim _{\tau \rightarrow \infty} \operatorname{dist}(T(\tau, t) z, A(\tau)),
\end{aligned}
$$

contradicting the fact that $z \in A^{*}(t)$ and proving that $T(t, s) A^{*}(s) \supset A^{*}(t)$.

We note that if $A_{0}=\left\{A_{0}(t): t \in \mathbb{R}\right\}, A_{1}=\left\{A_{1}(t): t \in \mathbb{R}\right\}, \cdots, A_{n}=\left\{A_{n}(t)\right.$ : $t \in \mathbb{R}\}$ are $n+1$ local attractors with $\varnothing=A_{0}(t) \subset A_{1}(t) \subset \cdots \subset A_{n}(t)=\mathscr{A}(t)$, for each $t \in \mathbb{R}$, then $\varnothing=A_{n}^{*}(t) \subset A_{n-1}^{*}(t) \subset \cdots \subset A_{0}^{*}(t)=\mathscr{A}(t)$, for each $t \in \mathbb{R}$.

Next we describe the construction of a Morse-decomposition for the pullback attractor of a generalized gradient-like evolution process relative to the disjoint set of isolated invariant families $\left\{\Xi_{1}, \cdots, \Xi_{n}\right\}$, and of the associated collection of increasing local attractors starting from the collection of isolated invariant sets. The following result plays a fundamental role on that.

Lemma 2.8. Let $\{T(t, s): t \geq s\}$ be a gradient-like evolution process with an associated disjoint set of isolated invariant families $\boldsymbol{\Xi}=\left\{\Xi_{1}, \cdots, \Xi_{n}\right\}$. Then, there exists $i \in\{1, \cdots, n\}$ such that $\Xi_{i}$ is a local attractor.

Proof. We first note that each $\Xi_{i}$ is an isolated invariant family which is not pinnedchain recurrent, and we need to show that there is some $i \in\{1, \cdots, n\}$ such that $W^{u}\left(\Xi_{i}\right)(t)=\Xi_{i}(t)$ for each $t \in \mathbb{R}$.

If that is not the case, for each $1 \leq i \leq n$ there is a global solution $\xi_{i}(t) \in \mathscr{A}(t)$ $\left(\right.$ with $\xi_{i}(s) \notin \Xi_{i}(s)$ for some $\left.s \in \mathbb{R}\right)$ such that $\lim _{t \rightarrow-\infty} \operatorname{dist}\left(\xi_{i}(t), \Xi_{i}(t)\right)=0$. Since $\xi_{i}(t)$ converges to some element of $\boldsymbol{\Xi}$ as $t \rightarrow \infty$, this necessarily produces a homoclinic structure and provides a contradiction with (H2).

Let $\{T(t, s): t \geq s\}$ be a gradient-like evolution process with the associated disjoint set of isolated invariant families $\boldsymbol{\Xi}=\left\{\Xi_{1}, \cdots, \Xi_{n}\right\}$. If (after possible reordering) $\Xi_{1}$ is a local attractor and $\Xi_{1}^{*}$ as in Definition [1.5 then we have that each $\Xi_{i}(s)$, for $i \geq 2$ and $s \in \mathbb{R}$, is contained in $\Xi_{1}^{*}(s)$ (also if $\xi: \mathbb{R} \rightarrow X$ is a global bounded solution and $\operatorname{dist}\left(\xi(t), \Xi_{i}(t)\right) \stackrel{t \rightarrow \infty}{\longrightarrow} 0$ with $i>1$, we have that $\xi(s) \in \Xi_{1}^{*}(s)$ for all $\left.s \in \mathbb{R}\right)$ and then for any $z \in \mathscr{A}(t) \backslash\left(\Xi_{1}(t) \cup \Xi_{1}^{*}(t)\right)$ and global solution $\xi: \mathbb{R} \rightarrow X$ with $\xi(s) \in \mathscr{A}(s)$, for each $s \in \mathbb{R}$, and $\xi(t)=z$ we have that

$$
\lim _{s \rightarrow-\infty} \operatorname{dist}\left(\xi(s), \Xi_{1}^{*}(s)\right)=0 \text { and } \lim _{s \rightarrow \infty} \operatorname{dist}\left(\xi(s), \Xi_{1}(s)\right)=0 .
$$

We can repeat the reasoning in Lemma 2.8 to conclude that there is $i \geq 2$ such that

$$
W^{u}\left(\Xi_{i}\right)(t) \cap \Xi_{1}^{*}(t)=\Xi_{i}(t) \text { for all } t .
$$

We relabel this isolated invariant family as $\Xi_{2}$ and define

$$
\Xi_{2,1}^{*}(t):=\left\{z \in \Xi_{1}^{*}(t): \operatorname{dist}\left(T(r+t, t) z, \Xi_{2}(r+t)\right) \nrightarrow 0 \text { as } r \rightarrow \infty\right\} .
$$

Then we have that, for each $t \in \mathbb{R}$ and $i=3, \cdots, n, \Xi_{i}(t) \subset \Xi_{2,1}^{*}(t)$ (also if $\xi: \mathbb{R} \rightarrow X$ is a global bounded solution and $\operatorname{dist}\left(\xi(t), \Xi_{i}(t)\right) \stackrel{t \rightarrow \infty}{\longrightarrow} 0$ with $i>2$, we have that $\xi(s) \in \Xi_{1}^{*}(s)$ for all $s \in \mathbb{R}$, and therefore $\xi(s) \in \Xi_{2,1}^{*}(s)$ for all $\left.s \in \mathbb{R}\right)$. As before, we can assume that $W^{u}\left(\Xi_{3}\right)(t) \cap \Xi_{2,1}^{*}(t)=\Xi_{3}(t)$ for each $t$ and define $\Xi_{3,2}^{*}$ in analogy to (2.1). 
Proceeding in this way until all isolated invariant families are exhausted, we obtain a reordering of $\boldsymbol{\Xi}=\left\{\Xi_{1}, \cdots, \Xi_{n}\right\}$ such that $\Xi_{1}$ is a local attractor for $\{T(t, s): t \geq s\}$, the set $\Xi_{1,0}^{*}:=\Xi_{1}^{*}$, and

$$
W^{u}\left(\Xi_{i}\right)(t) \cap \Xi_{i-1, i-2}^{*}(t)=\Xi_{i}(t) \text { for all } t \text { and } i=2, \cdots, n,
$$

where, for $i=2, \cdots, n$,

$$
\Xi_{i, i-1}^{*}(t):=\left\{z \in \Xi_{i-1, i-2}^{*}(t): \operatorname{dist}\left(T(r+t, t) z, \Xi_{i}(r+t)\right) \nrightarrow 0 \text { as } r \rightarrow \infty\right\} .
$$

Lemma 2.9. Let $\{T(t, s): t \geq s\}$ be a gradient-like evolution process with associated (reordered) disjoint set of isolated invariant families $\boldsymbol{\Xi}=\left\{\Xi_{1}, \cdots, \Xi_{n}\right\}$. Then, any global bounded solution $\xi: \mathbb{R} \rightarrow X$ satisfies

$$
\lim _{t \rightarrow-\infty} \operatorname{dist}\left(\xi(t), \Xi_{i}(t)\right)=0 \text { and } \lim _{t \rightarrow \infty} \operatorname{dist}\left(\xi(t), \Xi_{j}(t)\right),
$$

with $i \geq j$.

Proof. Indeed, if $j=1$ in (2.2) there is nothing to prove. If $j=2$ and (2.2) holds, we have that $\xi(t) \in \Xi_{1}^{*}(t)$ for all $t \in \mathbb{R}$, so if $1=i<j=2$, we have that $\xi(t) \in$ $W^{u}\left(\Xi_{1}\right)(t)=\Xi_{1}(t)$ for each $t$, which contradicts the fact that $\Xi_{1}^{*}(t) \cap \Xi_{1}(t)=\varnothing$ for each $t \in \mathbb{R}$. It follows that $i \geq j$.

For the general case, we suppose that $j \geq 3$ and that (2.2) holds. Then $\xi(t) \in$ $\Xi_{j-1, j-2}^{*}(t)$ for all $t$, so if $i<j$, we have that $\Xi_{j-1, j-2}^{*}(t) \subset \Xi_{i-1, i-2}^{*}(t)$ for all $t$ and $\xi(t) \in W^{u}\left(\Xi_{i}\right)(t) \cap \Xi_{i-1, i-2}^{*}(t)=\Xi_{i}(t)$ for every $t$. Thanks to the fact that the invariant families in $\boldsymbol{\Xi}$ are isolated, we must have $i=j$, which is a contradiction and proves the lemma.

We will prove that this reordering of $\left\{\Xi_{1}, \cdots, \Xi_{n}\right\}$ (which we denote the same) is a Morse-decomposition for $\{\mathscr{A}(t): t \in \mathbb{R}\}$ with a suitably chosen sequence $A_{0}(t) \subset A_{1}(t) \subset A_{2}(t) \subset \cdots \subset A_{n}(t)$ of local attractors:

For each $t \in \mathbb{R}$, define $A_{0}(t):=\varnothing, A_{1}(t):=\Xi_{1}(t)$, and for $j=2,3, \cdots, n$,

$$
A_{j}(t):=A_{j-1}(t) \cup W^{\mathrm{u}}\left(\Xi_{j}\right)(t)=\bigcup_{i=1}^{j} W^{\mathrm{u}}\left(\Xi_{i}\right)(t) .
$$

It is clear that $A_{n}(t)=\mathscr{A}(t)$.

Theorem 2.10. Let $\{T(t, s): t \geq s\}$ be a gradient-like process with a pullback attractor $\{\mathscr{A}(t): t \in \mathbb{R}\}$ and an associated disjoint set of isolated invariant families $\boldsymbol{\Xi}=\left\{\Xi_{1}, \cdots, \Xi_{n}\right\}$ reordered as explained above.

Assume that there exists $\delta>0$ such that, for $j=1,2, \cdots, n-1$ and $t \in \mathbb{R}$, it holds that

$$
\mathcal{O}_{\delta}\left(A_{j}(t)\right) \cap\left(\bigcup_{i=j+1}^{n} \Xi_{i}(t)\right)=\varnothing .
$$

Then, for each $j=0,1, \cdots, n$, the invariant family $A_{j}$ defined in (2.3) is a local attractor for $\{T(t, s): t \geq s\}$ and

$$
\Xi_{j}(t)=A_{j}(t) \cap A_{j-1}^{*}(t), \text { for all } t \in \mathbb{R} \text { and } 1 \leq j \leq n .
$$

Consequently, $\boldsymbol{\Xi}$ defines a Morse-decomposition for $\{\mathscr{A}(t): t \in \mathbb{R}\}$. 
Proof. Clearly $\left\{A_{j}(t): t \in \mathbb{R}\right\}$ is invariant and $A_{j}(t) \subset W^{u}\left(A_{j}\right)(t)$ for each $t \in$ $\mathbb{R}$. On the other hand, if $z \in W^{u}\left(A_{j}\right)(t)$, there is a global solution $\xi: \mathbb{R} \rightarrow$ $X$ with $\xi(t)=z$ and $\lim _{s \rightarrow-\infty} \operatorname{dist}\left(\xi(s), A_{j}(s)\right)=0$. Since $\{T(t, s): t \geq s\}$ is gradient-like and from (2.4) we have that there exists $k \in\{1,2, \cdots, j\}$ such that $\lim _{s \rightarrow-\infty} \operatorname{dist}\left(\xi(s), \Xi_{k}(s)\right)=0$. Therefore, $z \in W^{u}\left(\Xi_{k}\right)(t)$ with $k \leq j$. This implies that $z \in A_{j}$ and $W^{u}\left(A_{j}\right)(t) \subset A_{j}(t)$. Now we prove that $\left\{A_{j}(t): t \in \mathbb{R}\right\}$ is an isolated invariant family. Indeed, let $\delta>0$ be as in (2.4) and $\xi: \mathbb{R} \rightarrow X$ be a global solution with $\xi(t) \in \mathcal{O}_{\delta}\left(A_{j}(t)\right)$, for all $t \in \mathbb{R}$. Since $\{T(t, s): t \geq s\}$ is gradientlike, there is a $k \in\{1, \cdots, j\}$ such that $\operatorname{dist}\left(\xi(t), \Xi_{k}(t)\right) \stackrel{t \rightarrow-\infty}{\longrightarrow} 0$. It follows that $\xi(t) \in W^{u}\left(\Xi_{k}\right)(t) \subset A_{j}(t)$ for all $t \in \mathbb{R}$. This proves that $\left\{A_{j}(t): t \in \mathbb{R}\right\}$ is a local attractor.

It is easy to see that $\Xi_{j}(t) \subset A_{j}(t) \cap A_{j-1}^{*}(t)$. For the reverse inclusion, note that if $z \in A_{j}(t) \cap A_{j-1}^{*}(t)$, there exists a global solution $\xi: \mathbb{R} \rightarrow X$ with $\xi(t)=z$. Since $z \in A_{j}(t)$ we must have that $\lim _{s \rightarrow-\infty} \operatorname{dist}\left(\xi(s), \Xi_{k}(s)\right)$ for some $k \leq j$. As $\{T(t, s)$ : $t \geq s\}$ is gradient-like, there is $i \in\{1,2, \cdots, n\}$ such that $\lim _{s \rightarrow \infty} \operatorname{dist}\left(\xi(s), \Xi_{i}(s)\right)$ and, due to the fact that $z \in A_{j-1}^{*}(t)$, it follows that $i \geq j$. Since from Lemma 2.9 we must have that $i \leq k$, it follows that $k=i=j$, and again taking into account that $\{T(t, s): t \geq s\}$ is gradient-like, we have that $\xi(s) \in \Xi_{j}(s)$ for each $s \in \mathbb{R}$ and, in particular, $z=\xi(t) \in \Xi_{j}(t)$. This proves that $A_{j}(t) \cap A_{j-1}^{*}(t) \subset \Xi_{j}(t)$ and completes the proof.

Remark 2.11. Hypothesis (2.4) is not necessary in the autonomous case (see 1, Theorem 2.18]).

The following result plays a key role in the proof of the main results in this paper concerning the continuity of the Lyapunov function of a gradient-like evolution process. It extends Lemma 2.11 in [1] to the non-autonomous case.

Lemma 2.12. Let $\{T(t, s): t \geq s\}$ be an evolution process with a pullback attractor $\{\mathscr{A}(t): t \in \mathbb{R}\}$ and let $A=\{A(t): t \in \mathbb{R}\}$ be a local attractor which is not pinnedchain recurrent. Suppose that there exists $\varepsilon>0$ with

$$
\mathscr{A}(t) \cap \mathcal{O}_{\varepsilon}(A(t)) \cap \mathcal{O}_{\varepsilon}\left(A^{*}(t)\right)=\varnothing, \text { for all } t \in \mathbb{R} .
$$

Then, for each $\delta \in(0, \varepsilon)$ there is $\delta^{\prime} \in(0, \delta)$ such that

$$
T(t, s)\left(\mathscr{A}(s) \cap \mathcal{O}_{\delta^{\prime}}(A(s))\right) \subset \mathscr{A}(t) \cap \mathcal{O}_{\delta}(A(t)) \text { whenever } t \geq s .
$$

Proof. We argue by contradiction. Assume that there are $\delta>0$, sequences $\left(s_{j}\right)_{j \in \mathbb{N}}$, $\left(t_{j}\right)_{j \in \mathbb{N}}$ in $\mathbb{R}$, and $\left(x_{j}\right)_{j \in \mathbb{N}}$ in $X$ with $x_{j} \in \mathscr{A}\left(s_{j}\right)$ for each $j$, such that

$$
\begin{gathered}
s_{j} \leq t_{j}, \\
\operatorname{dist}\left(x_{j}, A\left(s_{j}\right)\right)<\frac{1}{j}, \text { but } \operatorname{dist}\left(T\left(t_{j}, s_{j}\right) x_{j}, A\left(t_{j}\right)\right) \geq \delta,
\end{gathered}
$$

for all $j \in \mathbb{N}$. Choose $j_{0} \in \mathbb{N}$ with $\operatorname{dist}\left(x_{j}, A\left(s_{j}\right)\right)<\frac{1}{j}<\varepsilon$ for all $j \geq j_{0}$. By the definition of a repeller, for each $j \geq j_{0}$ there is a $\tau_{j} \geq t_{j}$ such that

$$
\operatorname{dist}\left(T\left(\tau_{j}, s_{j}\right) x_{j}, A\left(\tau_{j}\right)\right)<\frac{1}{j} .
$$

It follows that $A$ is pinned-chain recurrent. This is a contradiction, and the lemma is proved. 
Remark 2.13. Note that the uniform separation property assumed in (2.5) is automatically satisfied in the autonomous case.

We can now show the following proposition:

Proposition 2.14. Let $A=\{A(t): t \in \mathbb{R}\}$ be an isolated invariant family for the evolution process $\{T(t, s): t \geq s\}$, with pullback attractor $\{\mathscr{A}(t): t \in \mathbb{R}\}$, such that $A(t) \subset \mathscr{A}(t)$ for all $t$, and for each $\delta>0$ there is $\delta^{\prime} \in(0, \delta)$ satisfying

$$
T(t, s)\left(\mathscr{A}(s) \cap \mathcal{O}_{\delta^{\prime}}(A(s))\right) \subset \mathscr{A}(t) \cap \mathcal{O}_{\delta}(A(t)) \text {, whenever } t \geq s .
$$

Then, $A=\{A(t): t \in \mathbb{R}\}$ is a local attractor for $\{T(t, s): t \geq s\}$, which is not pinned-chain recurrent.

Proof. Since $\{A(t): t \in \mathbb{R}\}$ is an isolated invariant family, there exists $\delta>0$ such that if $\xi: \mathbb{R} \rightarrow X$ is a global solution with $\xi(t) \in \mathcal{O}_{\delta}(A(t))$ for each $t \in \mathbb{R}$, then $\xi(t) \in A(t)$ for each $t \in \mathbb{R}$. Clearly $A(t) \subset W^{u}(A)(t)$ for each $t \in \mathbb{R}$, and if $\xi: \mathbb{R} \rightarrow X$ is a global solution such that $\lim _{s \rightarrow-\infty} \operatorname{dist}(\xi(s), A(s))=0$, choose $\delta^{\prime} \in(0, \delta)$ with

$$
T(t, s)\left(\mathscr{A}(s) \cap \mathcal{O}_{\delta^{\prime}}(A(s))\right) \subset \mathscr{A}(t) \cap \mathcal{O}_{\delta}(A(t)), \text { whenever } t \geq s .
$$

Choosing $s_{\delta} \in \mathbb{R}$ such that $\operatorname{dist}(\xi(s), A(s))<\delta^{\prime}$ for all $s \leq s_{\delta}$, we must have $\xi(t) \in$ $\mathcal{O}_{\delta}(A(t))$ for all $t \in \mathbb{R}$. Consequently, $\xi(t) \in A(t)$ for all $t \in \mathbb{R}$ and $W^{u}(A)(t) \subset A(t)$ for all $t \in \mathbb{R}$.

Finally, if $\{A(t): t \in \mathbb{R}\}$ is pinned-chain recurrent, there are $\delta>0$, sequences $\left(s_{j}\right)_{j \in \mathbb{N}},\left(t_{j}\right)_{j \in \mathbb{N}}$ and $\left(\tau_{j}\right)_{j \in \mathbb{N}}$ of real numbers, and a sequence $\left(x_{j}\right)_{j \in \mathbb{N}}$ in $X$, with $x_{j} \in \mathscr{A}\left(s_{j}\right)$ for each $j$, such that

$$
s_{j} \leq t_{j} \leq \tau_{j},
$$

$\operatorname{dist}\left(x_{j}, A\left(s_{j}\right)\right)<\frac{1}{j}, \quad \operatorname{dist}\left(T\left(t_{j}, s_{j}\right) x_{j}, A\left(t_{j}\right)\right) \geq \delta \quad$ and $\operatorname{dist}\left(T\left(\tau_{j}, s_{j}\right) x_{j}, A\left(\tau_{j}\right)\right)<\frac{1}{j}$.

That is clearly in contradiction with the fact that there exists $\delta^{\prime} \in(0, \delta)$ with

$$
T(t, s)\left(\mathscr{A}(s) \cap \mathcal{O}_{\delta^{\prime}}(A(s))\right) \subset \mathscr{A}(t) \cap \mathcal{O}_{\delta}(A(t)) \text { whenever } t \geq s,
$$

and the proof is complete.

Our next result is a first consequence of Lemma 2.12

Lemma 2.15. Let $\{T(t, s): t \geq s\}$ be an evolution process and $A=\{A(t): t \in \mathbb{R}\}$ be a local attractor which is not pinned-chain recurrent. Suppose that there exists $\varepsilon>0$ with

$$
\mathscr{A}(t) \cap \mathcal{O}_{\varepsilon}(A(t)) \cap \mathcal{O}_{\varepsilon}\left(A^{*}(t)\right)=\varnothing, \text { for all } t \in \mathbb{R} .
$$

If $K \subset \mathscr{A}(t)$ is compact and $K \cap A^{*}(t)=\varnothing$, then

$$
\lim _{\tau \rightarrow \infty} \operatorname{dist}(T(\tau, t) K, A(\tau))=0 .
$$

Proof. We prove the result by contradiction. Assume that there are $\delta>0$, a sequence $\left\{\tau_{j}\right\}_{j \in \mathbb{N}}$ in $\mathbb{R}$ with $\tau_{j} \stackrel{j \rightarrow \infty}{\longrightarrow} \infty$, and a sequence $\left\{x_{j}\right\}_{j \in \mathbb{N}}$ in $K$ with $x_{j} \stackrel{j \rightarrow \infty}{\longrightarrow}$ $x_{0} \in K$ such that

$$
\operatorname{dist}\left(T\left(\tau_{j}, t\right) x_{j}, A\left(\tau_{j}\right)\right) \geq \delta, \text { for all } j .
$$

Thanks to Lemma 2.12, we can choose $\delta^{\prime} \in(0, \delta)$ such that

$$
T(s, r) \mathcal{O}_{\delta^{\prime}}(A(r)) \subset \mathcal{O}_{\delta}(A(s)) \text { whenever } s \geq r .
$$


Thus, it follows from (2.6) that

$$
\operatorname{dist}\left(T(s, t) x_{j}, A(s)\right) \geq \delta^{\prime} \text { for all } s \in\left[t, \tau_{j}\right] \text { and all } j .
$$

Since $\tau_{j} \rightarrow \infty$, using (2.7), we have that

$$
\operatorname{dist}\left(T(s, t) x_{0}, A(s)\right) \geq \delta^{\prime} \text { for all } s \geq t .
$$

From the definition of $A^{*}(t)$ we have that $x_{0} \in A^{*}(t)$, which is in contradiction with $K \cap A^{*}(t)=\varnothing$, and the proof is therefore complete.

\section{A Lyapunov FunCtion For a Generalized GRAdIENT-LIKE PROCESS}

In this section we will prove that gradient-like evolution processes are gradient processes. A gradient evolution process is defined as follows:

Definition 3.1. We say that an evolution process $\{T(t, s): t \geq s\}$ with a pullback attractor $\{\mathscr{A}(t): t \in \mathbb{R}\}$ and a disjoint set of isolated invariant families $\boldsymbol{\Xi}=$ $\left\{\Xi_{1}, \cdots, \Xi_{n}\right\}$ is a gradient evolution process with respect to $\Xi$ if there is a function $V: \mathbb{R} \times X \rightarrow \mathbb{R}$ such that:

i) For each $z \in X$ and $t \in \mathbb{R}$, the function $[0, \infty) \ni r \mapsto V(r+t, T(r+t, t) z) \in \mathbb{R}$ is non-increasing.

ii) Given $t \in \mathbb{R}$ and $z \in \mathscr{A}(t), V(r+t, T(r+t, t) z)=V(t, z)$ for all $r \geq 0$ iff $z \in \bigcup_{i=1}^{n} \Xi_{i}(t)$ and $V\left(t, \Xi_{i}(t)\right)$ is a unitary set for each $t \in \mathbb{R}$ and $i \in\{1, \cdots, n\}$.

iii) For each $t \in \mathbb{R}$, the function $V_{t}: \mathscr{A}(t) \rightarrow \mathbb{R}$, given by $V_{t}(z):=V(t, z)$ for $z \in \mathscr{A}(t)$, is continuous.

A function $V: \mathbb{R} \times X \rightarrow \mathbb{R}$ with the properties above is called a Lyapunov function for the generalized gradient process $\{T(t, s): t \geq s\}$ with respect to $\boldsymbol{\Xi}$.

Before proving our main result, we need to establish the continuity of the invariant families $\left(A, A^{*}\right)$ of attractor-repeller pairs in the following sense:

Lemma 3.2. Let $\{T(t, s): t \geq s\}$ be a gradient-like evolution process in a metric space $X$ with a pullback attractor $\{\mathscr{A}(t): t \in \mathbb{R}\}$, and let $\left(A, A^{*}\right)$ be an attractorrepeller pair for $\{T(t, s): t \geq s\}$.

Then, for each $t_{0} \in \mathbb{R}$ we have

$$
\lim _{t \rightarrow t_{0}} \mathbf{d}_{\mathrm{H}}\left(A(t), A\left(t_{0}\right)\right)=0 \text { and } \lim _{t \rightarrow t_{0}} \mathbf{d}_{\mathrm{H}}\left(A^{*}(t), A^{*}\left(t_{0}\right)\right)=0 .
$$

Proof. In fact, we know that $A^{*}$ is invariant and then, by Lemma 2.10 and the proof of Theorem 2.9, both in [6], we obtain the conclusion (3.1).

Now, we can prove the main result in this paper.

Theorem 3.3. Let $\{T(t, s): t \geq s\}$ be an evolution process in a metric space $X$, with a pullback attractor $\{\mathscr{A}(t): t \in \mathbb{R}\}$, let $\left(A, A^{*}\right)$ be an attractor-repeller pair for $\{T(t, s): t \geq s\}$, and assume that $\left(A, A^{*}\right)$ is not pinned-chain recurrent. Suppose that there exists $\varepsilon>0$ with

$$
\mathcal{O}_{\varepsilon}(A(t)) \cap \mathcal{O}_{\varepsilon}\left(A^{*}(t)\right)=\varnothing, \text { for all } t \in \mathbb{R} .
$$

Then, there exists a function $k: \mathbb{R} \times X \rightarrow \mathbb{R}$ satisfying the following four properties:

i) For each $z \in X$ and $t \in \mathbb{R}$, the function $[0, \infty) \ni r \mapsto k(r+t, T(r+t, t) z) \in \mathbb{R}$ is non-increasing.

ii) If $k_{t}: X \rightarrow \mathbb{R}$ is defined by $k_{t}(z):=k(t, z)$, for each $t \in \mathbb{R}$ and $z \in X$, then $k_{t}^{-1}(0)=A(t)$ and $k_{t}^{-1}(1) \cap \mathscr{A}(t)=A^{*}(t)$. 
iii) Given $t \in \mathbb{R}$ and $z \in \mathscr{A}(t)$, if $k(r+t, T(r+t, t) z)=k(t, z)$ for all $r \geq 0$, then $z \in A(t) \cup A^{*}(t)$.

iv) $k_{t}: \mathscr{A}(t) \rightarrow \mathbb{R}$ is continuous, in $\mathscr{A}(t)$, for each $t \in \mathbb{R}$.

Proof. First, with the convention that $\operatorname{dist}(z, \varnothing)=1$, let $l: \mathbb{R} \times X \rightarrow[0,1]$ be the Uryshon function associated to the attractor-repeller pair $\left(A, A^{*}\right)$. That is, for each $t \in \mathbb{R}$ and $z \in X$,

$$
l(t, z):=\frac{\operatorname{dist}(z, A(t))}{\operatorname{dist}(z, A(t))+\operatorname{dist}\left(z, A^{*}(t)\right)} .
$$

We have that $l$ is well defined and that $l(\mathbb{R} \times X) \subset[0,1]$ is continuous in both variables $(t, z)$ and, for each $t \in \mathbb{R}$, is uniformly continuous in $X$ (that is, the family $\left\{l_{t}: X \rightarrow[0,1]: t \in \mathbb{R}\right\}$ is uniformly Lipschitz continuous, where $l_{t}: X \rightarrow \mathbb{R}$ is given by $l_{t}(z):=l(t, z)$, for each $t \in \mathbb{R}$ and $\left.z \in X\right)$. In fact, since, by (3.2),

$$
d_{0}:=\inf _{t \in \mathbb{R}}\left\{\inf \left\{\mathbf{d}(x, y): x \in A(t), y \in A^{*}(t)\right\}\right\} \geq \varepsilon>0,
$$

it holds that $|l(t, z)-l(t, w)| \leq \frac{2}{d_{0}} \mathbf{d}(z, w)$, for any $z, w \in X$ and $t \in \mathbb{R}$.

Now, from Lemma 3.2 it is not difficult to see that $l: \mathbb{R} \times X \rightarrow[0,1]$ is continuous in both variables. Moreover, it is easy to see that $l_{t}^{-1}(0)=A(t)$ and $l_{t}^{-1}(1)=A^{*}(t)$.

We define $k: \mathbb{R} \times X \rightarrow \mathbb{R}$ by

$$
k(t, z):=\sup _{r \geq 0} l(r+t, T(r+t, t) z) .
$$

We now show that $k: \mathbb{R} \times X \rightarrow \mathbb{R}$ has properties $i$ ) - iv) above.

Clearly, $k(\mathbb{R} \times X) \subset[0,1]$. To prove that, given $t \in \mathbb{R}$ and $z \in X$, the function $[0, \infty) \ni r \mapsto k(r+t, T(r+t, t) z) \in[0,1]$ is non-increasing, observe that, if $0 \leq$ $r_{1} \leq r_{2}$, we have

$$
\begin{aligned}
k\left(r_{2}+t, T\left(r_{2}+t, t\right) z\right) & =\sup _{r \geq 0} l\left(r+r_{2}+t, T\left(r+r_{2}+t, t\right) z\right) \\
& =\sup _{r \geq r_{2}} l(r+t, T(r+t, t) z) \\
& \leq \sup _{r \geq r_{1}} l(r+t, T(r+t, t) z) \\
& =\sup _{r \geq 0} l\left(r+r_{1}+t, T\left(r+r_{1}+t, t\right) z\right) \\
& =k\left(r_{1}+t, T\left(r_{1}+t, t\right) z\right) .
\end{aligned}
$$

By the definition of $k$ and from the invariance of $A$ and $A^{*}$, it is clear that $k_{t}(A(t))=\{0\}$ and $k_{t}\left(A^{*}(t)\right)=\{1\}$. Now, if $z \in X$ is such that $k(t, z)=0$, then $l(r+t, T(r+t, t) z)=0$ for all $r \geq 0$. In particular, $0=l(t, z)$, and then, $z \in A(t)$, that is, $k_{t}^{-1}(0) \subset A(t)$, which shows that $k_{t}^{-1}(0)=A(t)$. On the other hand, if $z \in$ $\mathscr{A}(t)$ is such that $k_{t}(z)=1$ and $z \notin A^{*}(t)$, then $\lim _{r \rightarrow \infty} \operatorname{dist}(T(r+t, t) z, A(r+t))=0$. From the definition of $l$ we obtain that $\lim _{r \rightarrow \infty} l(r+t, T(r+t, t) z)=0$. Therefore, there exists $r_{0}>0$ such that $1=k(t, z)=\sup _{0 \leq r \leq r_{0}} l(r+t, T(r+t, t) z)$. The continuity of $[0, \infty) \ni r \mapsto l(r+t, T(r+t, t) z) \in[0,1]$ implies the existence of $r^{\prime} \in\left[0, r_{0}\right]$ such that $l\left(r^{\prime}+t, T\left(r^{\prime}+t, t\right) z\right)=1$; that is, $T\left(r^{\prime}+t, t\right) z \in A^{*}\left(r^{\prime}+t\right)$ which contradicts the fact that $\lim _{r \rightarrow \infty} l(T(r+t, t) z, A(r+t))=0$. Thus, if $k(t, z)=1$ for 
some $t \in \mathbb{R}$ and $z \in \mathscr{A}(t)$, we must have that $z \in A^{*}(t)$. From this, we conclude that $k_{t}^{-1}(1) \cap \mathscr{A}(t) \subset A^{*}(t)$ and, consequently, $k_{t}^{-1}(1) \cap \mathscr{A}(t)=A^{*}(t)$.

We now prove that if $z \in \mathscr{A}(t)$ and $k(r+t, T(r+t, t) z)=k(t, z)$ for all $r \geq 0$, then $z \in A(t) \cup A^{*}(t)$. If $z \notin A(t) \cup A^{*}(t)$, then $\lim _{r \rightarrow \infty} \operatorname{dist}(T(r+t, t) z, A(r+t))=0$, and from the definition of $k$, we have that $k(t, z)=\lim _{r \rightarrow \infty} k(r+t, T(r+t, t) z)=0$. Since $k_{t}^{-1}(0)=A(t), z$ must belong to $A(t)$, which is a contradiction.

Next we prove the continuity of $k_{t}: \mathscr{A}(t) \rightarrow \mathbb{R}$. We split the proof into three cases:

Case 1). Continuity of $k_{t}: \mathscr{A}(t) \rightarrow \mathbb{R}$ in $A^{*}(t)$. Since $l(t, z) \leq k(t, z) \leq 1$, for all $t \in \mathbb{R}$ and $z \in X$, given $z_{0} \in A^{*}(t)$ and $z \in X$ we have

$$
\left|k(t, z)-k\left(t, z_{0}\right)\right|=1-k(t, z) \leq 1-l(t, z) .
$$

This and the continuity of $l: \mathbb{R} \times X \rightarrow \mathbb{R}$ in $\left(t, z_{0}\right)$ imply the continuity of $k_{t}: X \rightarrow \mathbb{R}$ in $z_{0}$.

Case 2). Continuity of $k_{t}: \mathscr{A}(t) \rightarrow \mathbb{R}$ in $A(t)$. From the equicontinuity of the family of functions $\left\{l_{t}: X \rightarrow \mathbb{R}: t \in \mathbb{R}\right\}$, given $\varepsilon>0$, there is $\delta>0$ such that $l_{s}\left(\mathcal{O}_{\delta}(A(s))\right) \subset[0, \varepsilon)$ for all $s \in \mathbb{R}$. Now, Lemma 2.12 implies that there exists $\delta^{\prime} \in(0, \delta)$ such that $T(r+t, t)\left(\mathscr{A}(t) \cap \mathcal{O}_{\delta^{\prime}}(A(t))\right) \subset \mathscr{A}(r+t) \cap \mathcal{O}_{\delta}(A(r+t))$, for all $r \geq 0$, from which we can conclude that $k_{t}\left(\mathscr{A}(t) \cap \mathcal{O}_{\delta^{\prime}}(A(t))\right) \subset[0, \varepsilon]$.

Case 3). Continuity of $k_{t}: \mathscr{A}(t) \rightarrow \mathbb{R}$ in $\mathscr{A}(t) \backslash\left(A(t) \cup A^{*}(t)\right)$. Given $z \in \mathscr{A}(t) \backslash(A(t)$ $\left.\cup A^{*}(t)\right)$, since $z \notin A^{*}(t)$, it follows that $\lim _{r \rightarrow \infty} \operatorname{dist}(T(r+t, t) z, A(r+t))=0$ and, since $z \notin A(t)$, it holds that $l(t, z)>0$. Choose $\delta>0$ such that $l_{s}\left(\mathcal{O}_{\delta}(A(s))\right) \subset$ $\left[0, \frac{l(t, z)}{2}\right)$ for all $s \in \mathbb{R}$ and, from Lemma 2.12. we can choose $\delta^{\prime} \in(0, \delta)$ such that $T(r+t, t)\left(\mathscr{A}(t) \cap \mathcal{O}_{\delta^{\prime}}(A(t))\right) \subset \mathscr{A}(r+t) \cap \mathcal{O}_{\delta}(A(r+t))$, for all $r \geq 0$.

From $\lim _{r \rightarrow \infty} \operatorname{dist}(T(r+t, t) z, A(r+t))=0$, there exists $\sigma>0$ with the property that $T(r+t, t) z \in \mathcal{O}_{\delta^{\prime}}(A(r+t))$, for all $r \geq \sigma$. From the continuity of $T(\sigma+t, t)$ : $X \rightarrow X$, there is a neighborhood $U_{1}$ of $z$ in $X$ such that $T(\sigma+t, t) U_{1} \subset \mathcal{O}_{\delta^{\prime}}(A(\sigma+t))$. Then, for all $w \in U_{1}$ we have that $T(\sigma+t, t) w \in \mathcal{O}_{\delta^{\prime}}(A(\sigma+t))$ so that $T(r+t, t) w \in$ $\mathcal{O}_{\delta}(A(r+t))$ for all $r \geq \sigma$ and $w \in U_{1} \cap \mathscr{A}(t)$.

Finally, from the continuity of $l_{t}$, let $U_{2}$ be a neighborhood of $z$ in $X$ such that $l(t, w)>\frac{l(t, z)}{2}$ for all $w \in U_{2}$ and write $U:=U_{1} \cap U_{2} \cap \mathscr{A}(t)$ so that, for all $w \in U$, it holds that $k(t, w)=\sup _{0<r<\sigma} l(r+t, T(r+t, t) w)$, from which we obtain the continuity of $k_{t}$ in points of $\mathscr{A}(t) \backslash\left(A(t) \cup A^{*}(t)\right)$.

The proof of our next result is very similar to the proof of Theorem 3.3 and follows the ideas in 11. It brings up a special case for which the continuity of the "Lyapunov functions" in both variables holds. We observe that the last theorem says that the "Lyapunov function" is continuous for each fixed $t$ and in the pullback attractor only.

Proposition 3.4. Let $\{T(t, s): t \geq s\}$ be an evolution process in a metric space $X$ with a pullback attractor $\{\mathscr{A}(t): t \in \mathbb{R}\}$, and let $\left(A, A^{*}\right)$ be an attractor-repeller 
pair for $\{T(t, s): t \geq s\}$ with

$$
\mathcal{O}_{\varepsilon_{0}}(A(t)) \cap \mathcal{O}_{\varepsilon_{0}}\left(A^{*}(t)\right)=\varnothing, \text { for all } t \in \mathbb{R},
$$

for some $\varepsilon_{0}>0$. Also assume that the following two conditions hold:

(a) The conclusion of Lemma 2.12 holds, considering neighborhoods of $A$ in $X$. That is, for each $\delta>0$ there is $\delta^{\prime} \in(0, \delta)$ such that

$$
T(r+t, t)\left(\mathcal{O}_{\delta^{\prime}}(A(t))\right) \subset \mathcal{O}_{\delta}(A(r+t)), \text { for all } r \geq 0 \text { and } t \in \mathbb{R} .
$$

(b) For each $t \in \mathbb{R}$ and $z \in X \backslash\left(A(t) \cup A^{*}(t)\right)$ we have

$$
\lim _{r \rightarrow \infty} \operatorname{dist}(T(r+t, t) z, A(r+t))=0 \text { or } \lim _{r \rightarrow \infty} \operatorname{dist}\left(T(r+t, t) z, A^{*}(r+t)\right)=0 .
$$

Then, the function $k: \mathbb{R} \times X \rightarrow \mathbb{R}$, defined in Theorem 3.3, is continuous (in both variables $(t, x)$ and for all $(t, x) \in \mathbb{R} \times X)$.

Remark 3.5. Hypotheses $(a)$ and $(b)$ are automatically satisfied in the autonomous case (see Lemma 2.11 and part (iii) of Lemma 2.13 in [1]).

Proof. Let us prove the continuity of $k$ in a point $\left(t_{0}, z_{0}\right) \in \mathbb{R} \times X$. As in the proof of Theorem 3.3. we consider three cases:

Case 1) $z_{0} \in A^{*}\left(t_{0}\right)$. Since $l(t, z) \leq k(t, z) \leq 1$, for all $t \in \mathbb{R}$ and $z \in X$ we can write

$$
\left|k(t, z)-k\left(t_{0}, z_{0}\right)\right|=1-k(t, z) \leq 1-l(t, z) .
$$

This and the continuity (in both variables) of $l: \mathbb{R} \times X \rightarrow \mathbb{R}$ in $\left(t_{0}, z_{0}\right)$ imply the continuity of $k: \mathbb{R} \times X \rightarrow \mathbb{R}$ in $\left(t_{0}, z_{0}\right)$.

Case 2) $z_{0} \in A\left(t_{0}\right)$. From

$$
|l(t, z)-l(t, w)| \leq \frac{2}{\varepsilon_{0}} \mathbf{d}(z, w), \quad \forall z, w \in X \text { and } t \in \mathbb{R},
$$

given $\varepsilon>0$, there is $\delta>0$ such that $l\left(s, \mathcal{O}_{\delta}(A(s))\right) \subset[0, \varepsilon)$ for all $s \in \mathbb{R}$.

On the other hand, from $(a)$ there exists $\delta^{\prime} \in(0, \delta)$ such that $T(r+t, t)\left(\mathcal{O}_{\delta^{\prime}}(A(t))\right)$ $\subset \mathcal{O}_{\delta}(A(r+t))$, for all $r \geq 0$ and $t \in \mathbb{R}$, from which we can conclude that $k\left(t, \mathcal{O}_{\delta^{\prime}}(A(t))\right) \subset[0, \varepsilon]$ for each $t \in \mathbb{R}$.

Now, by Lemma 3.2 let $\delta^{\prime \prime}>0$ be such that $\mathcal{O}_{\frac{\delta^{\prime}}{2}}\left(A\left(t_{0}\right)\right) \subset \mathcal{O}_{\delta^{\prime}}(A(t))$ whenever $\left|t-t_{0}\right|<\delta^{\prime \prime}$. Hence, for $\left|t-t_{0}\right|<\delta^{\prime \prime}$ and $\mathbf{d}\left(z, z_{0}\right)<\frac{\delta^{\prime}}{2}$ we have that $k(t, z) \leq \varepsilon$. This proves the continuity of $k: \mathbb{R} \times X \rightarrow \mathbb{R}$ in $\left(t_{0}, z_{0}\right)$ with $z_{0} \in A\left(t_{0}\right)$.

Case 3) $z_{0} \in X \backslash\left(A\left(t_{0}\right) \cup A^{*}\left(t_{0}\right)\right)$. By hypothesis $(b)$ we have $\lim _{r \rightarrow \infty} \operatorname{dist}\left(T\left(r+t_{0}, t_{0}\right) z_{0}, A\left(r+t_{0}\right)\right)=0$ or $\lim _{r \rightarrow \infty} \operatorname{dist}\left(T\left(r+t_{0}, t_{0}\right) z_{0}, A^{*}\left(r+t_{0}\right)\right)=0$.

First consider the case when $\lim _{r \rightarrow \infty} \operatorname{dist}\left(T\left(r+t_{0}, t_{0}\right) z_{0}, A\left(r+t_{0}\right)\right)=0$. Since $z_{0} \notin A^{*}\left(t_{0}\right)$ it holds that $l\left(t_{0}, z_{0}\right)>0$. Choose $\delta>0$ such that $l\left(s, \mathcal{O}_{\delta}(A(s))\right) \subset$ $\left[0, \frac{l\left(t_{0}, z_{0}\right)}{2}\right)$ for all $s \in \mathbb{R}$ and, from hyphotesis $(a)$, we choose $\delta^{\prime} \in(0, \delta)$ such that $T(r+t, t)\left(\mathcal{O}_{\delta^{\prime}}(A(t))\right) \subset \mathcal{O}_{\delta}(A(r+t))$, for all $r \geq 0$. From the fact that $\lim _{r \rightarrow \infty} \operatorname{dist}\left(T\left(r+t_{0}, t_{0}\right) z_{0}, A\left(r+t_{0}\right)\right)=0$, there exists $\sigma>0$ with the property that $T\left(\sigma+t_{0}, t_{0}\right) z_{0} \in \mathcal{O}_{\frac{\delta^{\prime}}{2}}\left(A\left(\sigma+t_{0}\right)\right)$. From the continuity of the process $\{T(t, s): t \geq s\}$ and Lemma 3.2, there is a neighborhood $U_{1}$ of $z_{0}$ in $X$ and $\delta^{\prime \prime}>0$ such that $T(\sigma+t, t) U_{1} \subset \mathcal{O}_{\frac{\delta^{\prime}}{2}}\left(A\left(\sigma+t_{0}\right)\right) \subset \mathcal{O}_{\delta^{\prime}}(A(\sigma+t))$ whenever $\left|t-t_{0}\right|<\delta^{\prime \prime}$. Then, for 
all $z \in U_{1}$ and $t \in\left(t_{0}-\delta^{\prime \prime}, t_{0}+\delta^{\prime \prime}\right)$, we have that $T(\sigma+t, t) z \in \mathcal{O}_{\delta^{\prime}}(A(\sigma+t))$ so that $T(r+t, t) z \in \mathcal{O}_{\delta}(A(r+t))$ for all $r \geq \sigma, z \in U_{1}$ and $t \in\left(t_{0}-\delta^{\prime \prime}, t_{0}+\delta^{\prime \prime}\right)$.

Now, from the continuity of $l: \mathbb{R} \times X \rightarrow \mathbb{R}$, let $U_{2}$ be a neighborhood of $z_{0}$ in $X$ and $0<\eta<\delta^{\prime \prime}$ such that $l(t, z)>\frac{l\left(t_{0}, z_{0}\right)}{2}$ for all $z \in U_{2}$ and $\left|t-t_{0}\right|<\eta$, and write $U:=U_{1} \cap U_{2}$ so that, for all $z \in U$ and $\left|t-t_{0}\right|<\eta$, it holds that $k(t, z)=\sup _{0 \leq r \leq \sigma} l(r+t, T(r+t, t) z)$, from which we obtain the continuity of $k$ in points $\left(t_{0}, z_{0}\right)$ with $z_{0} \in X \backslash\left(A\left(t_{0}\right) \cup A^{*}\left(t_{0}\right)\right)$ and $\lim _{r \rightarrow \infty} \operatorname{dist}\left(T\left(r+t_{0}, t_{0}\right) z_{0}, A\left(r+t_{0}\right)\right)=0$.

Now consider the case $\lim _{r \rightarrow \infty} \operatorname{dist}\left(T\left(r+t_{0}, t_{0}\right) z_{0}, A^{*}\left(r+t_{0}\right)\right)=0$. Hence $k\left(t_{0}, z_{0}\right)=$ 1 and, given $\varepsilon>0$, we can choose $\delta>0$ such that $l\left(s, \mathcal{O}_{\delta}\left(A^{*}(s)\right)\right) \subset(1-\varepsilon, 1]$ for all $s \in \mathbb{R}$. We choose $\sigma>0$ with $T\left(\sigma+t_{0}, t_{0}\right) z_{0} \in \mathcal{O}_{\frac{\delta}{2}}\left(A^{*}\left(\sigma+t_{0}\right)\right)$.

From the continuity of the process $\{T(t, s): t \geq s\}$ and Lemma 3.2. there is a neighborhood $U$ of $z_{0}$ in $X$ and $\delta^{\prime}>0$ such that $T(\sigma+t, t) U \subset \mathcal{O}_{\frac{\delta}{2}}\left(A^{*}\left(\sigma+t_{0}\right)\right) \subset$ $\mathcal{O}_{\delta}\left(A^{*}(\sigma+t)\right)$ whenever $\left|t-t_{0}\right|<\delta^{\prime}$. Therefore, $k(t, z) \geq l(t, z)>1-\varepsilon$ whenever $z \in U$ and $\left|t-t_{0}\right|<\delta^{\prime}$, and the proof of the proposition is complete.

Now, we wish to construct a continuous function $h: \mathbb{R} \times X \rightarrow \mathbb{R}$ which is nonincreasing along solutions and such that, given $t \in \mathbb{R}$ and $z \in X$, if $h(r+t, T(r+$ $t, t) z)=h(t, z)$ for all $r \geq 0$, then $z \in \mathcal{A}(t)$.

Lemma 3.6. Let $\{T(t, s): t \geq s\}$ be an evolution process in a metric space $X$, with a pullback attractor $\{\mathscr{A}(t): t \in \mathbb{R}\}$ satisfying the fact that for each $z \in X$ we have

$$
\lim _{r \rightarrow \infty} \operatorname{dist}(T(r+t, t) z, \mathscr{A}(r+t))=0,
$$

and for every $\delta>0$ there is $\delta^{\prime} \in(0, \delta)$ such that

$$
T(r+t, t)\left(\mathcal{O}_{\delta^{\prime}}(\mathscr{A}(t))\right) \subset \mathcal{O}_{\delta}(\mathscr{A}(r+t)), \text { for all } r \geq 0 \text { and } t \in \mathbb{R} .
$$

Then, the function $h: \mathbb{R} \times X \rightarrow \mathbb{R}$ given by

$$
h(t, z):=\sup _{r \geq 0} \operatorname{dist}(T(r+t, t) z, \mathscr{A}(r+t)),(t, z) \in \mathbb{R} \times X,
$$

is continuous (in both variables), and for each $(t, z) \in \mathbb{R} \times X$, the function $[0, \infty) \ni$ $r \mapsto h(r+t, T(r+t, t) z) \in \mathbb{R}$ is non-increasing with $h(t, z)=0$ iff $z \in \mathscr{A}(t)$.

Proof. Indeed, the proof of the fact that the function $[0, \infty) \ni r \mapsto h(r+t, T(r+$ $t, t) z) \in \mathbb{R}$ is non-increasing, for each $z \in X$ and $t \in \mathbb{R}$, is analogous to the proof of the same property for the function $k: \mathbb{R} \times X \rightarrow \mathbb{R}$ in Theorem 3.3 The proof of the continuity can be done in the same way as in cases 2) and 3) in the proof of the continuity of $k$ in Proposition 3.4 .

In the conditions of the last two results, using some ideas from 11, we can improve the conclusion of Theorem 3.3 by proving the following theorem.

Theorem 3.7. Let $\{T(t, s): t \geq s\}$ be an evolution process in a metric space $X$, with a pullback attractor $\{\mathscr{A}(t): t \in \mathbb{R}\}$ satisfying the fact that for each $z \in X$ we have

$$
\lim _{r \rightarrow \infty} \operatorname{dist}(T(r+t, t) z, \mathscr{A}(r+t))=0,
$$

and for every $\delta>0$ there is $\delta^{\prime} \in(0, \delta)$ such that

$$
T(r+t, t)\left(\mathcal{O}_{\delta^{\prime}}(\mathscr{A}(t))\right) \subset \mathcal{O}_{\delta}(\mathscr{A}(r+t)), \text { for all } r \geq 0 \text { and } t \in \mathbb{R} .
$$


Also, let $\left(A, A^{*}\right)$ be an attractor-repeller pair for $\{T(t, s): t \geq s\}$ with

$$
\mathcal{O}_{\varepsilon}(A(t)) \cap \mathcal{O}_{\varepsilon}\left(A^{*}(t)\right)=\varnothing, \text { for all } t \in \mathbb{R},
$$

for some $\varepsilon>0$ and, furthermore, satisfying the following conditions:

(a) The conclusion of Lemma 2.12 holds, considering neighborhoods of $A$ in $X$. That is, for each $\delta>0$ there is $\delta^{\prime} \in(0, \delta)$ such that

$$
T(r+t, t)\left(\mathcal{O}_{\delta^{\prime}}(A(t))\right) \subset \mathcal{O}_{\delta}(A(r+t)) \text {, for all } r \geq 0 \text { and } t \in \mathbb{R} \text {. }
$$

(b) For each $t \in \mathbb{R}$ and $z \in X \backslash\left(A(t) \cup A^{*}(t)\right)$ we have

$$
\lim _{r \rightarrow \infty} \operatorname{dist}(T(r+t, t) z, A(r+t))=0 \text { or } \lim _{r \rightarrow \infty} \operatorname{dist}\left(T(r+t, t) z, A^{*}(r+t)\right)=0 .
$$

Then, there exists a function $f: \mathbb{R} \times X \rightarrow \mathbb{R}$ satisfying the following four properties:

i) For each $(t, z) \in \mathbb{R} \times X$, the function $[0, \infty) \ni r \mapsto f(r+t, T(r+t, t) z) \in \mathbb{R}$ is non-increasing.

ii) If $t \in \mathbb{R}$ and $f_{t}: X \rightarrow \mathbb{R}$ is defined by $f_{t}(z):=f(t, z)$, for each $z \in X$, then $f_{t}^{-1}(0)=A(t)$ and $f_{t}^{-1}(1) \cap \mathscr{A}(t)=A^{*}(t)$.

iii) Given $t \in \mathbb{R}$ and $z \in X$, if $f(r+t, T(r+t, t) z)=f(t, z)$ for all $r \geq 0$, then $z \in A(t) \cup A^{*}(t)$.

iv) $f: \mathbb{R} \times X \rightarrow \mathbb{R}$ is continuous (in both variables).

Proof. In fact, let $k: \mathbb{R} \times X \rightarrow \mathbb{R}$ be the function given in Theorem 3.3 and $h: \mathbb{R} \times X \rightarrow \mathbb{R}$ be the function given in Lemma 3.6. We define $f: \mathbb{R} \times X \rightarrow \mathbb{R}$ by

$$
f(t, z):=k(t, z)+h(t, z),(t, z) \in \mathbb{R} \times X .
$$

It follows immediately from the properties of $h$ and $k$ that $f$ is continuous (in both variables) and that, for each $(t, z) \in \mathbb{R} \times X$, the function $[0, \infty) \ni r \mapsto$ $f(r+t, T(r+t, t) z) \in \mathbb{R}$ is non-increasing.

Proof of $i i)$. First, let $z \in A(t) \subset \mathscr{A}(t)$. Then $h(t, z)=0$ and, by Theorem 3.3. we have that $k(t, z)=0$. Hence $f(t, z)=0$ and $A(t) \subset f_{t}^{-1}(0)$. Conversely, if $z \in X$ is such that $f_{t}(z)=0$, we have that $h(t, z)=0$ and $k(t, z)=0$, therefore $z \in \mathscr{A}(t)$, and by $i i)$ in Theorem 3.3, $z \in A(t)$. This proves that $f_{t}^{-1}(0) \subset A(t)$. On the other hand, if $z \in A^{*}(t) \subset \mathscr{A}(t)$, we have that $h(t, z)=0$, and thus, $f(t, z)=$ $k(t, z)+h(t, z)=k(t, z)=1$ and $z \in A^{*}(t)$. It follows that $A^{*}(t) \subset f^{-1}(1) \cap \mathscr{A}(t)$. Now, let $z \in \mathscr{A}(t)$ with $f(t, z)=1$. It follows that $h(t, z)=0$ and $k(t, z)=1$. Therefore $z \in A^{*}(t)$ and the proof of $\left.i i\right)$ is complete.

Proof of $i i i)$. Let $(t, z) \in \mathbb{R} \times X$ with $f(r+t, T(r+t, t) z)=f(t, z)$ for all $r \geq 0$. If $z \in \mathscr{A}(t)$ we have $h(r+t, T(r+t, t) z)=0$ for all $r \geq 0$, and $k(r+t, T(r+t, t) z)=$ $k(t, z)$ for all $r \geq 0$, and, from iii) in Theorem 3.3, the conclusion follows. On the other hand, if $z \in X \backslash \mathscr{A}(t)$, then $\lim _{r \rightarrow \infty} \operatorname{dist}\left(T(r+t, t) z, A^{*}(r+t)\right)=0$. Indeed, if that was not the case, by assumption $\lim _{r \rightarrow \infty} \operatorname{dist}(T(r+t, t) z, A(r+t))=0$ it is easy to see that

$$
\begin{aligned}
f(t, z) & =\lim _{r \rightarrow \infty} f(r+t, T(r+t, t) z) \\
& =\lim _{r \rightarrow \infty} k(r+t, T(r+t, t) z)+\lim _{r \rightarrow \infty} h(r+t, T(r+t, t) z)=0 .
\end{aligned}
$$

Thus, $k(t, z)=0$ and $z \in A(t)$, which is in contradiction with the fact that $z \in$ $X \backslash \mathscr{A}(t)$. It follows that $\lim _{r \rightarrow \infty} \operatorname{dist}\left(T(r+t, t) z, A^{*}(r+t)\right)=0$. Now, using the same 
reasoning as in (3.5), we obtain

$$
\begin{aligned}
f(t, z) & =\lim _{r \rightarrow \infty} f(r+t, T(r+t, t) z) \\
& =\lim _{r \rightarrow \infty} k(r+t, T(r+t, t) z)+\lim _{r \rightarrow \infty} h(r+t, T(r+t, t) z)=1+0=1,
\end{aligned}
$$

but this cannot be true since the fact that $k(t, z) \geq k(r+t, T(r+t, t) z)$ for all $r \geq 0$ implies $1 \geq k(t, z) \geq \lim _{r \rightarrow \infty} k(r+t, T(r+t, t) z)=1$, that is, $k(t, z)=1$. Therefore $z \in A^{*}(t)$, contradicting $z \in X \backslash \mathscr{A}(t)$. This completes the proof.

Remark 3.8. We note that the function $h$ plays a fundamental role in the proof of iii).

Finally, analogously to Theorem 3.4 in [1, joining all of the results in this section, the following result holds.

Theorem 3.9. Let $\{T(t, s): t \geq s\}$ be a gradient-like evolution process with respect to the isolated invariant families $\boldsymbol{\Xi}=\left\{\Xi_{1}, \cdots, \Xi_{n}\right\}$, reordered in the way described in Theorem 2.10, and with a pullback attractor $\{\mathscr{A}(t): t \in \mathbb{R}\}$.

Let $A_{j}(t)=\bigcup_{i=1}^{j} W^{u}\left(\Xi_{i}\right)(t)$, for $1 \leq j \leq n$ and $t \in \mathbb{R}$.

Assume that the following conditions hold:

$(S 1)$ There exists $\delta>0$ such that $\mathcal{O}_{\varepsilon}\left(A_{j}(t)\right) \cap \mathcal{O}_{\varepsilon}\left(A_{j}^{*}(t)\right)=\varnothing$, for all $1 \leq j \leq n$ and $t \in \mathbb{R}$.

(S2) Given $\delta>0$, there is $\delta^{\prime} \in(0, \delta)$ such that

$$
T(r+t, t)\left(\mathscr{A}(t) \cap \mathcal{O}_{\delta^{\prime}}\left(A_{j}(t)\right)\right) \subset \mathscr{A}(t+r) \cap \mathcal{O}_{\delta}\left(A_{j}(r+t)\right),
$$

for all $r \geq 0,1 \leq j \leq n$ and $t \in \mathbb{R}$.

Then, there exists a Lyapunov function $V: \mathbb{R} \times X \rightarrow \mathbb{R}$ with properties $i$ )-iii) of Definition 3.1, and such that $V\left(t, \Xi_{k}(t)\right)=\{k-1\}$, for all $t \in \mathbb{R}$ and $k=1, \cdots, n$.

Moreover, if $(S 2)$ holds for neighborhoods of the $A_{j}$ 's in $X$ and given $(t, z) \in$ $\mathbb{R} \times X$ and $1 \leq j \leq n, \lim _{r \rightarrow \infty} \operatorname{dist}\left(T(r+t, t) z, A_{j}(r+t) \cup A_{j}^{*}(r+t)\right)=0$, then the Lyapunov function $V: \mathbb{R} \times X \rightarrow \mathbb{R}$ can be chosen continuous in both variables.

\section{Non-Autonomous PERTURBations OF AUTONOMOUS EVOLUTION PROCESSES}

Now, we will exhibit a class of examples to which our previous abstract theory can be applied. This class consists of the non-autonomous perturbations of gradient-like semigroups.

To begin with, we recall Theorem 3.9 in [5]:

Theorem 4.1. Let $X$ be a metric space and, for each $\eta \in[0,1]$, let $\left\{T_{\eta}(t, s): t \geq s\right\}$ be an evolution process on $X$ with a pullback attractor $\left\{\mathscr{A}_{\eta}(t): t \in \mathbb{R}\right\}$. Assume that the following conditions hold:

(a) $\bigcup_{\eta \in[0,1]} \bigcup_{t \in \mathbb{R}} \mathscr{A}_{\eta}(t)$ is compact.

(b) $\left\{T_{0}(t, s): t \geq s\right\}$ is an autonomous evolution process and $\left\{T_{0}(t, 0)=S(t)\right.$ : $t \geq 0\}$ is a gradient-like semigroup relative to the set of equilibria $\mathcal{E}_{0}=$ $\left\{y_{1,0}^{*}, \cdots, y_{n, 0}^{*}\right\}$. 
(c) For each $\eta \in(0,1],\left\{T_{\eta}(t, s): t \geq s\right\}$ possesses $n$ isolated global solutions $\xi_{i, \eta}^{*}: \mathbb{R} \rightarrow X, i=1,2, \cdots, n, \eta \in(0,1]$, and $\lim _{\eta \rightarrow 0+} \sup _{1 \leq i \leq n} \sup _{t \in \mathbb{R}} \mathbf{d}\left(\xi_{i, \eta}^{*}(t), y_{i, 0}^{*}\right)=$ 0 .

(d) For each compact set $K \subset \mathbb{R}^{+} \times X$,

$$
\lim _{\eta \rightarrow 0^{+}} \sup _{s \in \mathbb{R}} \sup _{(t, x) \in K} \mathbf{d}\left(T_{\eta}(t+s, s) x, T_{0}(t+s, s) x\right)=0 .
$$

(e) There exist $\mu>0$ and $\eta_{1} \in(0,1]$ such that, if $\xi_{\eta}: \mathbb{R} \rightarrow X$ is a bounded solution of $\left\{T_{\eta}(t, s): t \geq s\right\}$ with $\eta \leq \eta_{1}$ so that there exists $t_{0} \in \mathbb{R}$ and some $i \in$ $\{1, \cdots, n\}$ with $\sup \operatorname{dist}\left(\xi_{\eta}(t), \xi_{i, \eta}^{*}(\mathbb{R})\right)<\mu\left(\right.$ resp. $\sup \operatorname{dist}\left(\xi_{\eta}(t), \xi_{i, \eta}^{*}(\mathbb{R})\right)$ $<\mu)$, then $\lim _{t \rightarrow-\infty} \mathbf{d}\left(\xi_{\eta}(t), \xi_{i, \eta}^{*}(t)\right)=0$ (resp. $\left.\lim _{t \rightarrow \infty} \mathbf{d}\left(\xi_{\eta}(t), \xi_{i, \eta}^{*}(t)\right)=0\right)$.

Then, there exists $\eta_{0} \in\left(0, \eta_{1}\right]$ such that, for each $\eta \in\left(0, \eta_{0}\right],\left\{T_{\eta}(t, s): t \geq s\right\}$ is a non-autonomous gradient-like evolution process with respect to the disjoint set of isolated invariant families $\mathcal{E}_{\eta}=\left\{\xi_{1, \eta}^{*}, \cdots, \xi_{n, \eta}^{*}\right\}$.

The following lemma will be crucial to establish our main results in this section (see Lemma 3.1 in [7] for the proof).

Lemma 4.2. Let $X$ be a metric space and $\left\{T_{\eta}(t, s): t \geq s\right\}_{\eta \in[0,1]}$ be a family of evolution processes on $X$ with pullback attractors $\left\{\mathscr{A}_{\eta}(t): t \in \mathbb{R}\right\}_{\eta \in[0,1]}$. Assume that conditions $(a),(b)$ and $(d)$ of Theorem 4.1 are satisfied.

Let $\left\{\eta_{k}\right\}_{k \in \mathbb{N}}$ be a sequence in $(0, \infty)$ with $\eta_{k} \stackrel{k \rightarrow \infty}{\longrightarrow} 0,\left\{t_{k}\right\}_{k \in \mathbb{N}}$ be a sequence in $\mathbb{R}$ and $\left\{\xi_{k}\right\}_{k \in \mathbb{N}}$ be a sequence in $C(\mathbb{R}, X)$ such that, for each $k \in \mathbb{N}, \xi_{k}: \mathbb{R} \rightarrow X$ is a bounded global solution for $\left\{T_{\eta_{k}}(t, s): t \geq s\right\}$.

Then, there is a subsequence of $\left\{\xi_{k}\right\}_{k \in \mathbb{N}}$, which we denote the same, and a global solution $\xi: \mathbb{R} \rightarrow \mathscr{A}_{0}$ for $\left\{T_{0}(t, 0)=S(t): t \geq 0\right\}$ such that

$$
\lim _{k \rightarrow \infty} \sup _{|t| \leq R} \mathbf{d}\left(\xi_{k}\left(t+t_{k}\right), \xi(t)\right)=0, \text { for each } R>0 .
$$

Let $X$ be a metric space and, for each $\eta \in[0,1],\left\{T_{\eta}(t, s): t \geq s\right\}$ an evolution process on $X$ with a pullback attractor $\left\{\mathscr{A}_{\eta}(t): t \in \mathbb{R}\right\}$ which satisfies conditions (a)-(e) in Theorem 4.1. We suppose that the set of equilibria, $\mathcal{E}_{0}=\left\{y_{1,0}^{*}, \cdots, y_{n, 0}^{*}\right\}$, of $\left\{T_{0}(t, 0)=S(t): t \geq 0\right\}$ is reordered so that it is a Morse-decomposition of the global attractor $\mathscr{A}_{0}$, as in Theorem 2.10 (see also Theorem 2.17 in [1] for the autonomous framework).

For $\eta \in[0,1]$, reordering the $\mathcal{E}_{\eta}=\left\{\xi_{1, \eta}^{*}, \cdots, \xi_{n, \eta}^{*}\right\}$ so that $\left.\sup _{1 \leq i \leq n t \in \mathbb{R}} \sup _{1 \leq \xi_{i, \eta}^{*}}(t), y_{i, 0}^{*}\right)$ $\stackrel{\eta \rightarrow 0^{+}}{\longrightarrow} 0$, let $A_{0, \eta}(t):=\varnothing, A_{1, \eta}(t):=\left\{\xi_{1, \eta}^{*}(t)\right\}$ and

$$
A_{j, \eta}(t):=A_{j-1, \eta}(t) \cup W^{u}\left(\xi_{j, \eta}^{*}\right)(t), j=2, \cdots, n,
$$

for each $t \in \mathbb{R}$. Also, for each $\eta \in[0,1], 1 \leq j \leq n$ and $t \in \mathbb{R}$, let

$$
A_{j, \eta}^{*}(t):=\left\{z \in \mathscr{A}_{\eta}(t): \operatorname{dist}\left(T_{\eta}(r+t, t) z, A_{j, \eta}(r+t)\right) \nrightarrow 0 \text { as } r \rightarrow \infty\right\} .
$$

If we assume these constructions, we can prove the following results:

Lemma 4.3. Consider a metric space $X$ and, for each $\eta \in[0,1],\left\{T_{\eta}(t, s): t \geq s\right\}$ an evolution process on $X$ with a pullback attractor $\left\{\mathscr{A}_{\eta}(t): t \in \mathbb{R}\right\}$ which satisfies conditions $(a)-(e)$ in Theorem 4.1. Assume that $\mathcal{E}_{0}=\left\{y_{1,0}^{*}, \cdots, y_{n, 0}^{*}\right\}$ is reordered 
so that it is a Morse-decomposition for $\left\{T_{0}(t, 0)=S(t): t \geq 0\right\}$ and that $\mathcal{E}_{\eta}$ is reordered in such a way that $\lim _{\eta \rightarrow 0+} \sup _{1 \leq i \leq n} \sup _{t \in \mathbb{R}} \mathbf{d}\left(\xi_{i, \eta}^{*}(t), y_{i, 0}^{*}\right)=0$.

Then, the families of $A_{j, \eta}$ and $A_{j, \eta}^{*}$ defined by (4.1) and (4.2) behave upper semicontinuously as $\eta \rightarrow 0^{+}$, that is,

$$
\lim _{\eta \rightarrow 0^{+}} \sup _{t \in \mathbb{R}} \operatorname{dist}\left(A_{j, \eta}(t), A_{j, 0}\right)=0
$$

and

$$
\lim _{\eta \rightarrow 0^{+}} \sup _{t \in \mathbb{R}} \operatorname{dist}\left(A_{j, \eta}^{*}(t), A_{j, 0}^{*}\right)=0
$$

for each $j=1,2, \cdots, n$.

Proof. Indeed, thanks to the fact that $A_{j, 0}$ and $A_{j, 0}^{*}$ are disjoint compact sets (see [1]) for all $j$, we can pick $\varepsilon>0$ such that, for all $j=1,2, \cdots, n$,

$$
\mathcal{O}_{\varepsilon}\left(A_{j, 0}\right) \cap \mathcal{O}_{\varepsilon}\left(A_{j, 0}^{*}\right)=\varnothing .
$$

First, we will prove that $\lim _{\eta \rightarrow 0^{+}} \max _{1 \leq j \leq n} \sup _{t \in \mathbb{R}} \operatorname{dist}\left(A_{j, \eta}(t), A_{j, 0}\right)=0$. If this is not true, there are $\delta \in(0, \varepsilon), j \in\{1,2, \cdots, n\}$, a sequence $\left\{\eta_{k}\right\}_{k \in \mathbb{N}}$ in $(0,1]$ with $\eta_{k} \stackrel{k \rightarrow \infty}{\longrightarrow} 0^{+}$, a sequence $\left\{\tau_{k}\right\}_{k \in \mathbb{N}}$ in $\mathbb{R}$ and a sequence $\left\{x_{k}\right\}_{k \in \mathbb{N}}$ in $X$ such that, for each $k \in \mathbb{N}$, $x_{k} \in A_{j, \eta_{k}}\left(\tau_{k}\right)$ and

$$
\operatorname{dist}\left(x_{k}, A_{j, 0}\right) \geq \delta .
$$

Now, by the definition of $A_{j, \eta}(t)$, we can assume that there is $i \leq j$, fixed, such that for each natural $k$, there is a global solution $\xi_{k}: \mathbb{R} \rightarrow X$ for $\left\{T_{\eta_{k}}(t, s): t \geq s\right\}$ with $\xi_{k}\left(\tau_{k}\right)=x_{k}$ and

$$
\lim _{t \rightarrow-\infty} \mathbf{d}\left(\xi_{k}(t), \xi_{i, \eta_{k}}^{*}(t)\right)=0 .
$$

On the other hand, by hypothesis $(c)$, it follows the existence of $\eta_{\delta}>0$ such that, for all $\eta \leq \eta_{\delta}$,

$$
\sup _{t \in \mathbb{R}} \operatorname{dist}\left(\xi_{i, \eta}^{*}(t), A_{j, 0}\right)<\frac{\delta}{2} .
$$

Consequently, by (4.6) and (4.7), for each $k$ there exists $t_{k} \in \mathbb{R}$ such that

$$
\operatorname{dist}\left(\xi_{k}(t), A_{j, 0}\right)<\delta \text { for all } t<t_{k}
$$

and

$$
\operatorname{dist}\left(\xi_{k}\left(t_{k}\right), A_{j, 0}\right)=\delta .
$$

Thus, we define $\widetilde{\xi}_{k}: \mathbb{R} \rightarrow X$ by $\widetilde{\xi}_{k}(t):=\xi_{k}\left(t+t_{k}\right), t \in \mathbb{R}$, and, by Lemma 4.2. we can assume that there is a global solution $\xi: \mathbb{R} \rightarrow X$ for the semigroup $\left\{T_{0}(t, 0)=S(t): t \geq 0\right\}$ such that, for each $R>0$,

$$
\lim _{k \rightarrow \infty} \sup _{|t| \leq R} \mathbf{d}\left(\widetilde{\xi}_{k}(t), \xi(t)\right)=0 .
$$

By (4.8), we have that $\operatorname{dist}\left(\xi(t), A_{j, 0}\right) \leq \delta$ for all $t \leq 0$, so, by (4.5), we must have $\xi(0) \in A_{j, 0}$, but (4.9) means that $\operatorname{dist}\left(\xi(0), A_{j, 0}\right)=\delta$. This contradiction proves (4.3).

Now, we prove that $\lim _{\eta \rightarrow 0^{+}} \max _{1 \leq j \leq n_{t \in \mathbb{R}}} \operatorname{dist}\left(A_{j, \eta}^{*}(t), A_{j, 0}^{*}\right)=0$. If it does not hold, there exist $\delta \in(0, \varepsilon), j=1,2, \cdots, n$, a sequence $\left\{\eta_{k}\right\}_{k \in \mathbb{N}}$ in $(0,1]$ with $\eta_{k} \stackrel{k \rightarrow \infty}{\longrightarrow} 0$, 
a sequence $\left\{\tau_{k}\right\}_{k \in \mathbb{N}}$ in $\mathbb{R}$, and a sequence $\left\{x_{k}\right\}_{k \in \mathbb{N}}$ in $X$ such that, for each $k \in \mathbb{N}$, $x_{k} \in A_{j, \eta_{k}}^{*}\left(\tau_{k}\right)$ and

$$
\operatorname{dist}\left(x_{k}, A_{j, 0}^{*}\right) \geq \delta
$$

Now, by the definition of $A_{j, \eta}^{*}(t)$ (and Theorem 4.1), we can assume that there is $i \geq j+1$, fixed, such that for each $k \in \mathbb{N}$, there is a global solution $\xi_{k}: \mathbb{R} \rightarrow X$ for $\left\{T_{\eta_{k}}(t, s): t \geq s\right\}$ with $\xi_{k}\left(\tau_{k}\right)=x_{k}$ and

$$
\lim _{t \rightarrow \infty} \mathbf{d}\left(\xi_{k}(t), \xi_{i, \eta_{k}}^{*}(t)\right)=0 .
$$

On the other hand, hyphotesis $(c)$ implies the existence of $\eta_{\delta}^{*}>0$ such that, for all $\eta \leq \eta_{\delta}^{*}$

$$
\sup _{t \in \mathbb{R}} \operatorname{dist}\left(\xi_{i, \eta}^{*}(t), A_{j, 0}^{*}\right)<\frac{\delta}{2},
$$

whence, by (4.10) and (4.11), for each $k \in \mathbb{N}$, there exists $t_{k} \in \mathbb{R}$ such that

$$
\operatorname{dist}\left(\xi_{k}(t), A_{j, 0}^{*}\right)<\delta \text { for all } t>t_{k}
$$

and

$$
\operatorname{dist}\left(\xi_{k}\left(t_{k}\right), A_{j, 0}^{*}\right)=\delta \text {. }
$$

Thus, we define $\widetilde{\xi}_{k}: \mathbb{R} \rightarrow X$ by $\widetilde{\xi}_{k}(t):=\xi_{k}\left(t+t_{k}\right), t \in \mathbb{R}$, and, by Lemma 4.2. we can assume that there is a global solution $\xi: \mathbb{R} \rightarrow X$ for the semigroup $\left\{T_{0}(t, 0)=S(t): t \geq 0\right\}$ such that, for each $R>0$,

$$
\lim _{k \rightarrow \infty} \sup _{|t| \leq R} \mathbf{d}\left(\widetilde{\xi}_{k}(t), \xi(t)\right)=0 .
$$

From (4.12) we have that $\operatorname{dist}\left(\xi(t), A_{j, 0}^{*}\right) \leq \delta$ for all $t>0$ and, as a consequence of (4.5), we must have $\xi(0) \in A_{j, 0}^{*}$. On the other hand, (4.13) implies that $\operatorname{dist}\left(\xi(0), A_{j, 0}^{*}\right)=\delta$. This is a contradiction and (4.4) is proved, completing the proof of the lemma.

Lemma 4.4. Let $X$ be a metric space and, for each $\eta \in[0,1],\left\{T_{\eta}(t, s): t \geq s\right\}$ an evolution process on $X$ with a pullback attractor $\left\{\mathscr{A}_{\eta}(t): t \in \mathbb{R}\right\}$ which satisfies conditions $(a)-(e)$ in Theorem 4.1. Assume that $\mathcal{E}_{0}=\left\{y_{1,0}^{*}, \cdots, y_{n, 0}^{*}\right\}$ is reordered in such a way that it is a Morse-decomposition for $\left\{T_{0}(t, 0)=S(t): t \geq 0\right\}$ and that $\mathcal{E}_{\eta}$ is reordered in such a way that $\lim _{\eta \rightarrow 0+} \sup _{1 \leq i \leq n} \sup _{t \in \mathbb{R}} \mathbf{d}\left(\xi_{i, \eta}^{*}(t), y_{i, 0}^{*}\right)=0$.

If the families of local unstable sets $\left\{W_{\eta, \rho}^{u}\left(\xi_{j}^{*}\right)(t)\right\}_{\eta \in[0,1]}$ behave lower semicontinuously, then the families of $A_{j, \eta}$ behave lower semicontinuously as $\eta \rightarrow 0^{+}$; that $i s$,

$$
\lim _{\eta \rightarrow 0^{+}} \sup _{t \in \mathbb{R}} \operatorname{dist}\left(A_{j, 0}, A_{j, \eta}(t)\right)=0 .
$$

If the families of local stable sets $\left\{W_{\eta, \rho}^{s}\left(\xi_{j}^{*}\right)(t) \cap \mathscr{A}_{\eta}(t)\right\}_{\eta \in[0,1]}$ behave lower semicontinuously and $T_{\eta}(t, s): A(s) \rightarrow A(t)$ is injective for each $t \geq s, \eta \in[0,1]$, then

$$
\lim _{\eta \rightarrow 0^{+}} \sup _{t \in \mathbb{R}} \operatorname{dist}\left(A_{j, 0}^{*}, A_{j, \eta}(t)^{*}\right)=0 .
$$

Proof. It follows analogously to the proof of Theorem 2.13 in [6]. 
Next we exhibit an example (pictorial) for which we have lower semicontinuity of repellers but for which we do not have structural stability. The purpose of this example is to show that the systems which satisfy the hypothesis imposed in the last part of Lemma 4.4 are a larger class than that of the structurally stable systems.

Below, the figure labelled 01.a corresponds to the perturbed attractor-repeller pair $\left(A_{i, \eta}, A_{i, \eta}^{*}\right)$ with $A_{i, \eta}$ pictured in black and $A_{i, \eta}^{*}$ pictured in red, $0 \leq i \leq 5$. The figure labelled 01.b corresponds to the limiting attractor-repeller $\left(A_{i}, A_{i}^{*}\right)$ pair with $A_{i}$ pictured in black and $A_{i}^{*}$ pictured in red, $1 \leq i \leq 5$. Of course $A_{5, \eta}\left(A_{0, \eta}^{*}\right)$ and $A_{5}\left(A_{0}^{*}\right)$ correspond to the global attractor, whereas $A_{5, \eta}^{*}\left(A_{0, \eta}\right)$ and $A_{5}^{*}\left(A_{0}\right)$ correspond to the empty set. Color can be seen online.

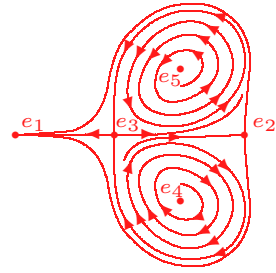

Figure 00.a
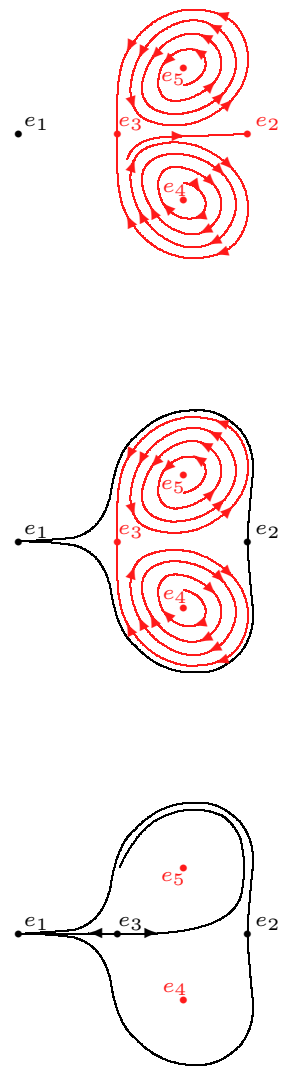

Figure 01.a

Figure 02.a

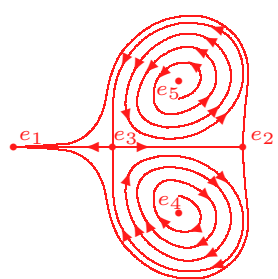

Figure 00.b

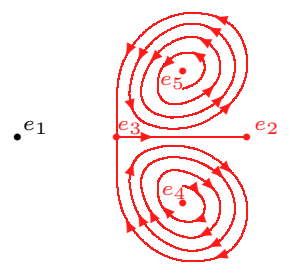

Figure 01.b

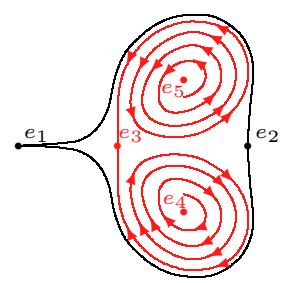

Figure 02.b

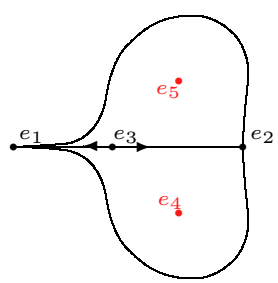

Figure 03.b 


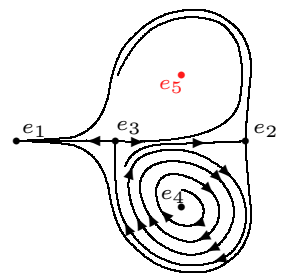

Figure 04.a

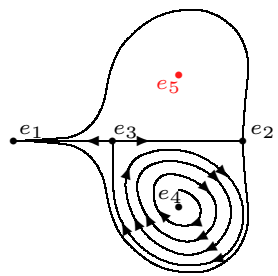

Figure 04.b

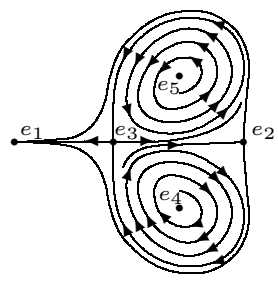

Figure 05.a

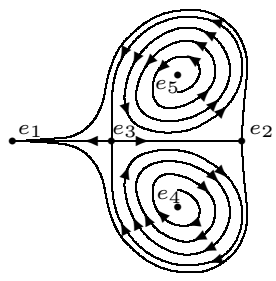

Figure 05.b

Proposition 4.5. Consider a metric space $X$ and, for each $\eta \in[0,1],\left\{T_{\eta}(t, s)\right.$ : $t \geq s\}$ an evolution process on $X$ with a pullback attractor $\left\{\mathscr{A}_{\eta}(t): t \in \mathbb{R}\right\}$ which satisfies conditions $(a)-(e)$ in Theorem 4.1. Assume that $\mathcal{E}_{0}=\left\{y_{1,0}^{*}, \cdots, y_{n, 0}^{*}\right\}$ is reordered so that it is a Morse-decomposition for $\left\{T_{0}(t, 0)=S(t): t \geq 0\right\}$ and that $\mathcal{E}_{\eta}$ is reordered in such a way that $\lim _{\eta \rightarrow 0+} \sup _{1 \leq i \leq n} \sup _{t \in \mathbb{R}} \mathbf{d}\left(\xi_{i, \eta}^{*}(t), y_{i, 0}^{*}\right)=0$.

Then, there are $\varepsilon>0$ and $\eta_{0}>0$ such that

$$
\mathcal{O}_{\varepsilon}\left(A_{j, \eta}(t)\right) \cap \mathcal{O}_{\varepsilon}\left(\bigcup_{i=j+1}^{n}\left\{\xi_{i, \eta}^{*}(t)\right\}\right)=\varnothing
$$

for each $j=1,2, \cdots, n-1, t \in \mathbb{R}$ and $\eta \in\left[0, \eta_{0}\right]$.

Proof. Indeed, by Theorem 2.17 in 1, we can take $\varepsilon>0$ such that, for each $j=1,2, \cdots, n-1$ and $t \in \mathbb{R}$,

$$
\mathcal{O}_{2 \varepsilon}\left(A_{j, 0}\right) \cap \mathcal{O}_{2 \varepsilon}\left(\bigcup_{i=j+1}^{n}\left\{y_{i, 0}^{*}\right\}\right)=\varnothing .
$$

We note that the proposition will be accomplished if we show the following stronger conditions:

For each $\delta \in(0, \varepsilon]$ there is $\eta_{\delta}>0$ such that

$$
A_{j, \eta}(t) \subset \mathcal{O}_{\delta}\left(A_{j, 0}\right)
$$

and

$$
\bigcup_{i=j+1}^{n}\left\{\xi_{i, \eta}^{*}(t)\right\} \subset \mathcal{O}_{\delta}\left(\bigcup_{i=j+1}^{n}\left\{y_{i, 0}^{*}\right\}\right)
$$

for all $t \in \mathbb{R}, j=1,2, \cdots, n-1$ and $\eta \in\left[0, \eta_{\delta}\right]$. 
Clearly (4.16) follows from hyphotesis $(c)$, and (4.15) is a direct consequence of (4.3) in Lemma 4.3. This completes the proof of the proposition.

Using Proposition 4.5 we can show that the sets $A_{j, \eta}$, defined above, are all local attractors for all suitable $\eta$. This is proved in the following proposition.

Proposition 4.6. Let $X$ be a metric space and, for each $\eta \in[0,1],\left\{T_{\eta}(t, s): t \geq s\right\}$ an evolution process on $X$ with a pullback attractor $\left\{\mathscr{A}_{\eta}(t): t \in \mathbb{R}\right\}$ which satisfies conditions (a)-(e) from Theorem 4.1. Assume that $\mathcal{E}_{0}=\left\{y_{1,0}^{*}, \cdots, y_{n, 0}^{*}\right\}$ is reordered so that it is a Morse-decomposition for $\left\{T_{0}(t, 0)=S(t): t \geq 0\right\}$ and that $\mathcal{E}_{\eta}$ is reordered in such a way that $\lim _{\eta \rightarrow 0+} \sup _{1 \leq i \leq n} \sup _{t \in \mathbb{R}} \mathbf{d}\left(\xi_{i, \eta}^{*}(t), y_{i, 0}^{*}\right)=0$.

Then, there exists $\eta_{0}>0$ such that the invariant family $A_{j, \eta}:=\left\{A_{j, \eta}(t)\right.$ : $t \in \mathbb{R}\}$ is a local attractor for the evolution process $\left\{T_{\eta}(t, s): t \geq s\right\}$, for each $j=1,2, \cdots, n$ and $\eta \in\left(0, \eta_{0}\right]$.

Proof. Indeed, by Lemma 4.3 and the proof of Proposition 4.5, we can choose $\varepsilon>0$, $\delta \in(0, \varepsilon]$ and $\eta_{0}>0$ such that

$$
A_{j, \eta}(t) \subset \mathcal{O}_{\delta}\left(A_{j, 0}\right)
$$

and

$$
\bigcup_{i=j+1}^{n}\left\{\xi_{i, \eta}^{*}(t)\right\} \subset \mathcal{O}_{\delta}\left(\bigcup_{i=j+1}^{n}\left\{y_{i, 0}^{*}\right\}\right)
$$

for each $j=1,2, \cdots, n-1, t \in \mathbb{R}$ and $\eta \in\left[0, \eta_{0}\right]$, where $\varepsilon>0$ satisfies

$$
\mathcal{O}_{2 \varepsilon}\left(A_{j, 0}\right) \cap \mathcal{O}_{2 \varepsilon}\left(\bigcup_{i=j+1}^{n}\left\{y_{i, 0}^{*}\right\}\right)=\varnothing .
$$

Thus, if $\Gamma_{j, \eta}$ is the trace of the family $A_{j, \eta}$, it follows that $\Gamma_{j, \eta} \subset \mathcal{O}_{\varepsilon}\left(A_{j, 0}\right)$ for $j=1,2, \cdots, n-1$ and $\eta \in\left[0, \eta_{0}\right]$, and therefore

$$
\mathcal{O}_{\varepsilon}\left(\Gamma_{j, \eta}\right) \cap \mathcal{O}_{\varepsilon}\left(\bigcup_{i=j+1}^{n}\left\{\xi_{i, \eta}^{*}(t)\right\}\right)=\varnothing
$$

for each $j=1,2, \cdots, n-1, t \in \mathbb{R}$ and $\eta \in\left[0, \eta_{0}\right]$.

Now, for a fixed $\eta \in\left[0, \eta_{0}\right]$, let $\xi_{\eta}: \mathbb{R} \rightarrow X$ be a global solution for $\left\{T_{\eta}(t, s)\right.$ : $t \geq s\}$ with $\xi_{\eta}(t) \in \mathcal{O}_{\varepsilon}\left(\Gamma_{j, \eta}\right)$ for all $t \in \mathbb{R}$ and, recalling that $\left\{T_{\eta}(t, s): t \geq s\right\}$ is a gradient-like evolution process with respect to $\mathcal{E}_{\eta}=\left\{\xi_{1, \eta}^{*}, \cdots, \xi_{n, \eta}^{*}\right\}$ for $\eta \in\left[0, \eta_{0}\right]$ (by Theorem 4.1), let $i \in\{1,2, \cdots, n\}$ such that

$$
\lim _{t \rightarrow-\infty} \mathbf{d}\left(\xi_{\eta}(t), \xi_{i, \eta}^{*}(t)\right)=0 .
$$

By (4.17), we must have $i \leq j$, so $\xi_{\eta}(t) \in W^{u}\left(\xi_{i, \eta}^{*}\right)(t) \subset A_{j, \eta}(t)$ for all $t \in \mathbb{R}$, which tells us that the family $A_{j, \eta}=\left\{A_{j, \eta}(t): t \in \mathbb{R}\right\}$ is invariant and isolated for each $j=1,2, \cdots, n$ and $\eta \in\left[0, \eta_{0}\right]$.

On the other hand, let $\xi_{\eta}: \mathbb{R} \rightarrow X$ be a global solution for $\left\{T_{\eta}(t, s): t \geq s\right\}$ with $\eta \in\left[0, \eta_{0}\right]$ and $\lim _{t \rightarrow-\infty} \operatorname{dist}\left(\xi_{\eta}(t), A_{j, \eta}(t)\right)=0$. If $t_{0}$ is such that $\operatorname{dist}\left(\xi_{\eta}(t), A_{j, \eta}(t)\right)<$ $\varepsilon$ for $t \leq t_{0}$, as $\mathcal{O}_{\varepsilon}\left(A_{j, \eta}(t)\right) \cap \mathcal{O}_{\varepsilon}\left(\bigcup_{i=j+1}^{n}\left\{\xi_{i, \eta}^{*}(t)\right\}\right)=\varnothing$ for all $t \in \mathbb{R}$, using the above reasoning, we must have $\xi_{\eta}(t) \in A_{j, \eta}(t)$ for all $t \in \mathbb{R}$, which shows that 
$W^{u}\left(A_{j, \eta}\right)(t) \subset A_{j, \eta}(t)$ for each $t \in \mathbb{R}$. Now, the inclusion $A_{j, \eta}(t) \subset W^{u}\left(A_{j, \eta}\right)(t)$, for each $t \in \mathbb{R}$, holds thanks to the invariance of the family $A_{j, \eta}$, showing that $W^{u}\left(A_{j, \eta}\right)(t)=A_{j, \eta}(t)$ for all $t \in \mathbb{R}$, and completing the proof of the proposition.

The separation property between the local attractor and its repeller is also satisfied for the class of the non-autonomous perturbation of a gradient-like semigroup. This is what we will show in the next proposition.

Proposition 4.7. Let $X$ be a metric space and, for each $\eta \in[0,1],\left\{T_{\eta}(t, s): t \geq s\right\}$ an evolution process on $X$ with a pullback attractor $\left\{\mathscr{A}_{\eta}(t): t \in \mathbb{R}\right\}$ which satisfies conditions $(a)-(e)$ in Theorem 4.1. Assume that $\mathcal{E}_{0}=\left\{y_{1,0}^{*}, \cdots, y_{n, 0}^{*}\right\}$ is reordered so that it is a Morse-decomposition for $\left\{T_{0}(t, 0): t \geq 0\right\}$ and that $\mathcal{E}_{\eta}$ is reordered in such a way that $\lim _{\eta \rightarrow 0+} \sup _{1 \leq i \leq n} \sup _{t \in \mathbb{R}} \mathbf{d}\left(\xi_{i, \eta}^{*}(t), y_{i, 0}^{*}\right)=0$. Consider the families of local attractors and corresponding repellers defined in (4.1) and (4.2).

Then, there exist $\varepsilon>0$ and $\eta_{0}>0$ such that, for all $t \in \mathbb{R}, j=1,2, \cdots, n$ and $\eta \in\left[0, \eta_{0}\right]$, we have

$$
\mathcal{O}_{\varepsilon}\left(A_{j, \eta}(t)\right) \cap \mathcal{O}_{\varepsilon}\left(A_{j, \eta}^{*}(t)\right)=\varnothing .
$$

Proof. Indeed, thanks to the fact that $A_{j, 0}$ and $A_{j, 0}^{*}$ are disjoint compact sets (see [1]) for all $j$, we can choose $\varepsilon>0$ such that, for all $j=1,2, \cdots, n$,

$$
\mathcal{O}_{2 \varepsilon}\left(A_{j, 0}\right) \cap \mathcal{O}_{2 \varepsilon}\left(A_{j, 0}^{*}\right)=\varnothing .
$$

By Lemma 4.3 for each $\delta \in(0, \varepsilon]$, there is $\eta_{\delta}>0$ such that

$$
A_{j, \eta}(t) \subset \mathcal{O}_{\delta}\left(A_{j, 0}\right)
$$

and

$$
A_{j, \eta}^{*}(t) \subset \mathcal{O}_{\delta}\left(A_{j, 0}^{*}\right),
$$

for every $\eta \in\left[0, \eta_{\delta}^{*}\right], t \in \mathbb{R}$ and $j=1,2, \cdots, n$. The result now follows.

Theorem 4.8. Let $X$ be a metric space and, for each $\eta \in[0,1],\left\{T_{\eta}(t, s): t \geq s\right\}$ a nonlinear evolution process on $X$ with a pullback attractor $\left\{\mathscr{A}_{\eta}(t): t \in \mathbb{R}\right\}$ which satisfies conditions $(a)-(e)$ of Theorem 4.1]. Assume that $\mathcal{E}_{0}=\left\{y_{1,0}^{*}, \cdots, y_{n, 0}^{*}\right\}$ is reordered so that it is a Morse-decomposition for $\left\{T_{0}(t, 0): t \geq 0\right\}$ and that $\mathcal{E}_{\eta}$ is reordered in such a way that $\lim _{\eta \rightarrow 0+} \sup _{1 \leq i \leq n} \sup _{t \in \mathbb{R}} \mathbf{d}\left(\xi_{i, \eta}^{*}(t), y_{i, 0}^{*}\right)=0$. Also consider the families of local attractors $A_{j, \eta}=\left\{A_{j, \eta}(t): t \in \mathbb{R}\right\}$ defined in (4.1) and their associated repellers $A_{j, \eta}^{*}=\left\{A_{j, \eta}^{*}(t): t \in \mathbb{R}\right\}$ given by (4.2).

Then, there exists $\eta_{0}>0$ such that for all $\eta \in\left(0, \eta_{0}\right], t \in \mathbb{R}$ and $j=1,2, \cdots, n$,

$$
A_{j, \eta}(t) \cap A_{j-1, \eta}^{*}(t)=\left\{\xi_{j, \eta}^{*}(t)\right\} .
$$

That is, the set $\mathcal{E}_{\eta}=\left\{\xi_{1, \eta}^{*}, \cdots, \xi_{n, \eta}^{*}\right\}$ determines a Morse decomposition of the pullback attractor $\left\{\mathscr{A}_{\eta}(t): t \in \mathbb{R}\right\}$ for $\left\{T_{\eta}(t, s): t \geq s\right\}$ for each $\eta \in\left(0, \eta_{0}\right]$. In particular, such Morse-decomposition behaves continuously.

Proof. Indeed, it is clear that $\xi_{j, \eta}^{*}(t) \in W^{u}\left(\xi_{j, \eta}^{*}\right)(t) \subset A_{j, \eta}(t)$ for each $1 \leq j \leq n$, $t \in \mathbb{R}$ and $\eta \in[0,1]$. Now, using Proposition 4.5] let $\varepsilon>0$ and $\eta_{0}>0$ be such that, for all $t \in \mathbb{R}, 2 \leq j \leq n$ and $\eta \in\left[0, \eta_{0}\right]$, we have

$$
\mathcal{O}_{2 \varepsilon}\left(A_{j-1, \eta}(t)\right) \cap \mathcal{O}_{2 \varepsilon}\left(\bigcup_{i=j}^{n}\left\{\xi_{i, \eta}^{*}(t)\right\}\right)=\varnothing .
$$


From (4.20), $\operatorname{dist}\left(T_{\eta}(r+t, t) \xi_{j, \eta}^{*}(t), A_{j-1, \eta}(t+r)\right)=\operatorname{dist}\left(\xi_{j, \eta}^{*}(t+r), A_{j-1, \eta}(t+r)\right) \geq$ $\varepsilon$ for all $r \geq 0$. Hence $\xi_{j, \eta}^{*}(t) \in A_{j-1, \eta}^{*}(t)$ for all $t \in \mathbb{R}$. Therefore, $\left\{\xi_{j, \eta}^{*}(t)\right\} \subset$ $A_{j, \eta}(t) \cap A_{j-1, \eta}^{*}(t)$ for every $\eta \in\left(0, \eta_{0}\right], t \in \mathbb{R}$ and $1 \leq j \leq n$.

Conversely, we note that, by Lemma 4.3 , given $\delta \in(0, \varepsilon]$ there exists $\eta_{\delta}>0$ such that for all $\eta \in\left(0, \eta_{\delta}\right], t \in \mathbb{R}$ and $1 \leq j \leq n$ we have that

$$
A_{j, \eta}(t) \cap A_{j-1, \eta}^{*}(t) \subset \mathcal{O}_{\frac{\delta}{2}}\left(A_{j, 0}\right) \cap \mathcal{O}_{\frac{\delta}{2}}\left(A_{j-1,0}^{*}\right) .
$$

Now, if the statement

$A_{j, \eta}(t) \cap A_{j-1, \eta}^{*}(t) \subset\left\{\xi_{j, \eta}^{*}(t)\right\}$ for all $t \in \mathbb{R}, 1 \leq j \leq n$ and for all $\eta>0$ suitably small

does not hold, there are $j \in\{1, \cdots, n\}$, a sequence $\left\{\eta_{k}\right\}_{k \in \mathbb{N}}$ in $(0,1]$ with $\eta_{k} \rightarrow 0^{+}$, a sequence $\left\{t_{k}\right\}_{k \in \mathbb{N}}$ in $\mathbb{R}$, and a sequence $\left\{z_{k}\right\}_{k \in \mathbb{N}}$ in $X$ with $z_{k} \in A_{j, \eta_{k}}\left(t_{k}\right) \cap$ $A_{j-1, \eta_{k}}^{*}\left(t_{k}\right) \backslash\left\{\xi_{j, \eta_{k}}^{*}\left(t_{k}\right)\right\}$ for all $k \in \mathbb{N}$. Hence, for each $k \in \mathbb{N}$, there exists a global solution $\xi_{k}: \mathbb{R} \rightarrow X$ for $\left\{T_{\eta_{k}}(t, s): t \geq s\right\}$ with $\xi_{k}\left(t_{k}\right)=z_{k}$, and we can assume that there are $i \leq j$ and $l \geq j$, fixed, with

$$
\lim _{t \rightarrow-\infty} \mathbf{d}\left(\xi_{k}(t), \xi_{i, \eta_{k}}^{*}(t)\right)=0 \text { and } \lim _{t \rightarrow \infty} \mathbf{d}\left(\xi_{k}(t), \xi_{l, \eta_{k}}^{*}(t)\right)=0 .
$$

Moreover, $\xi_{k}(t) \in \mathcal{O}_{\frac{\delta}{2}}\left(A_{j, 0}\right) \cap \mathcal{O}_{\frac{\delta}{2}}\left(A_{j-1,0}^{*}\right)$ for each $k \in \mathbb{N}$ and $t \in \mathbb{R}$. Therefore, if we choose, for suitably large $k, \tau_{k}$ and $\sigma_{k}$ with

$$
\mathbf{d}\left(\xi_{k}(t), y_{i, 0}^{*}\right)<\frac{\delta}{2} \text { for } t \leq \tau_{k} \text { and } \mathbf{d}\left(\xi_{k}(t), y_{l, 0}^{*}\right)<\frac{\delta}{2} \text { for } t \geq \sigma_{k},
$$

we must have $y_{i, 0}^{*}, y_{l, 0}^{*} \in \mathcal{O}_{\delta}\left(A_{j, 0}\right) \cap \mathcal{O}_{\delta}\left(A_{j-1,0}^{*}\right)$. Then, by (4.20), $i=l=j$; that is, $y_{i, 0}^{*}=y_{l, 0}^{*}=y_{j, 0}^{*}$. Thus $\xi_{i, \eta_{k}}^{*}=\xi_{l, \eta_{k}}^{*}=\xi_{j, \eta_{k}}^{*}$, which means that the solution $\xi_{k}: \mathbb{R} \rightarrow X$ is a homoclinic solution (because $z_{k} \neq \xi_{j, \eta_{k}}^{*}\left(t_{k}\right)$ for every $k)$, contradicting the fact that $\left\{T_{\eta_{k}}(t, s): t \geq s\right\}$ is gradient-like with respect to $\mathcal{E}_{\eta_{k}}=\left\{\xi_{1, \eta_{k}}^{*}, \cdots, \xi_{n, \eta_{k}}^{*}\right\}$, completing the proof.

Definition 4.9. We say that the family $\left\{T_{\eta}(t, s): t \geq s\right\}_{\eta \in[0,1]}$ of evolution processes in a metric space $X$ is collectively asymptotically compact if $\left\{T_{\eta_{k}}\left(t_{k}+\right.\right.$ $\left.\left.\tau_{k}, \tau_{k}\right) x_{k}\right\}_{k \in \mathbb{N}}$ is relatively compact whenever $\left\{\eta_{k}\right\}_{k \in \mathbb{N}}$ is a sequence in $(0,1],\left\{x_{k}\right\}_{k \in \mathbb{N}}$ is a bounded sequence in $X,\left\{t_{k}\right\}_{k \in \mathbb{N}}$ is a sequence in $\mathbb{R}^{+}$and $\left\{\tau_{k}\right\}_{k \in \mathbb{N}}$ is a sequence in $\mathbb{R}$ with $\eta_{k} \stackrel{k \rightarrow \infty}{\longrightarrow} 0$ and $t_{k} \stackrel{k \rightarrow \infty}{\longrightarrow} \infty$.

Lemma 4.10. Let $\left\{T_{\eta}(t, s): t \geq s\right\}_{\eta \in[0,1]}$ be a family of evolution processes in a metric space $X$ with a corresponding family of pullback attractors $\left\{\mathscr{A}_{\eta}\right\}_{\eta \in[0,1]}:=$ $\left\{\mathscr{A}_{\eta}(t): t \in \mathbb{R}\right\}_{\eta \in[0,1]}$, which satisfies conditions $(a)$ and $(d)$ in Theorem 4.1]. Assume that the family $\left\{T_{\eta}(t, s): t \geq s\right\}_{\eta \in[0,1]}$ is collectively asymptotically compact and that $T_{0}(t, s)=S(t-s)$ for all $t \geq s$, where $\{S(t): t \geq 0\}$ is a semigroup.

Let $\left\{A_{\eta}\right\}_{\eta \in[0,1]}:=\left\{A_{\eta}(t): t \in \mathbb{R}\right\}_{\eta \in[0,1]}$ be such that $A_{\eta}(t) \subset \mathscr{A}_{\eta}(t)$ for every $t \in$ $\mathbb{R}$ with $A_{0}$ a local attractor for the semigroup $\{S(t): t \geq 0\}$ (that is, $\omega\left(\mathcal{O}_{\varepsilon}\left(A_{0}\right)\right)=$ $A_{0}$ for some $\left.\varepsilon>0\right)$.

If $\left\{A_{\eta}\right\}_{\eta \in[0,1]}$ is continuous at $\eta=0$, that is,

$$
\lim _{\eta \rightarrow 0^{+}} \sup _{t \in \mathbb{R}} \mathbf{d}_{H}\left(A_{\eta}(t), A_{0}\right)=0
$$

then, given $\delta \in(0, \varepsilon)$, there exist $\delta^{\prime} \in(0, \delta)$ and $\eta_{\delta}>0$ such that, for all $\eta \in\left[0, \eta_{\delta}\right]$, it holds that

$$
T_{\eta}(t, s)\left(\mathcal{O}_{\delta^{\prime}}\left(A_{\eta}(s)\right)\right) \subset \mathcal{O}_{\delta}\left(A_{\eta}(t)\right) \text { whenever } t \geq s
$$


Proof. If not, there are $\delta>0$ and sequences $\left\{z_{j}\right\}_{j \in \mathbb{N}}$ in $X,\left\{\eta_{j}\right\}_{j \in \mathbb{N}}$ in $(0,1],\left\{t_{j}\right\}_{j \in \mathbb{N}}$ in $\mathbb{R}^{+}$and $\left\{\tau_{j}\right\}_{j \in \mathbb{N}}$ in $\mathbb{R}$ such that $\eta_{j} \stackrel{j \rightarrow \infty}{\longrightarrow} 0^{+}, t_{j} \stackrel{j \rightarrow \infty}{\longrightarrow} \infty, \operatorname{dist}\left(z_{j}, A_{\eta_{j}}\left(\tau_{j}\right)\right)<\frac{1}{j}$ for all $j$,

$$
\operatorname{dist}\left(T_{\eta_{j}}\left(t+\tau_{j}, \tau_{j}\right) z_{j}, A_{\eta_{j}}\left(t+\tau_{j}\right)\right)<\delta \text { for all } t \in\left[0, t_{j}\right), j \in \mathbb{N},
$$

and

$$
\operatorname{dist}\left(T_{\eta_{j}}\left(t_{j}+\tau_{j}, \tau_{j}\right) z_{j}, A_{\eta_{j}}\left(t_{j}+\tau_{j}\right)\right)=\delta \text { for all } j \in \mathbb{N} \text {. }
$$

If, for each $j$, we now define $\xi_{j}:\left[-t_{j}, \infty\right) \rightarrow X$ by $\xi_{j}(t):=T_{\eta_{j}}\left(t+t_{j}+\tau_{j}, \tau_{j}\right) z_{j}$, from the collective asymptotic compactness and the uniform convergence in compact sets (hypothesis $(d)$ ), it is not difficult to see that there exists a bounded global solution $\xi_{0}: \mathbb{R} \rightarrow X$ for $\left\{T_{0}(t, 0)=S(t): t \geq 0\right\}$ and a subsequence of $\left\{\xi_{j}\right\}_{j \in \mathbb{N}}$, denoted the same, such that for all $t, \xi_{0}(t)=\lim _{j \rightarrow \infty} \xi_{j}(t)$.

On the other hand, given $t<0$, for all $j$ large enough, it holds that

$$
\operatorname{dist}\left(\xi_{j}(t), A_{0}\right) \leq \operatorname{dist}\left(\xi_{j}(t), A_{\eta_{j}}\left(t+\tau_{j}\right)\right)+\operatorname{dist}\left(A_{\eta_{j}}\left(t+\tau_{j}\right), A_{0}\right),
$$

from where, by the upper-semicontinuity of $\left\{A_{\eta}\right\}_{\eta \in[0,1]}$, we obtain that for all $t<0$,

$$
\operatorname{dist}\left(\xi_{0}(t), A_{0}\right) \leq \delta,
$$

and from $\delta=\operatorname{dist}\left(\xi_{j}(0), A_{\eta_{j}}\left(t_{j}+\tau_{j}\right)\right) \leq \operatorname{dist}\left(\xi_{j}(0), A_{0}\right)+\operatorname{dist}\left(A_{0}, A_{\eta_{j}}\left(t_{j}+\tau_{j}\right)\right)$, by the lower-semicontinuity of $\left\{A_{\eta}\right\}_{\eta \in[0,1]}$, it follows that $\operatorname{dist}\left(\xi_{0}(0), A_{0}\right)=\delta$.

But, as $\delta<\varepsilon$, then $A_{0}$ attracts $K=\left\{\xi_{0}(t): t \leq 0\right\}$, which contradicts the fact that $\operatorname{dist}\left(\xi_{0}(0), A_{0}\right)=\delta$.

Proposition 4.11. Let $X$ be a metric space and, for each $\eta \in[0,1],\left\{T_{\eta}(t, s)\right.$ : $t \geq s\}$ an evolution process on $X$ with a pullback attractor $\left\{\mathscr{A}_{\eta}(t): t \in \mathbb{R}\right\}$ which satisfies conditions $(a)-(e)$ in Theorem 4.1. Assume that $\mathcal{E}_{0}=\left\{y_{1,0}^{*}, \cdots, y_{n, 0}^{*}\right\}$ is reordered so that it is a Morse-decomposition for $\left\{T_{0}(t, 0): t \geq 0\right\}$ and that $\mathcal{E}_{\eta}$ is reordered in such a way that $\lim _{\eta \rightarrow 0+} \sup _{1 \leq i \leq n} \sup _{t \in \mathbb{R}} \mathbf{d}\left(\xi_{i, \eta}^{*}(t), y_{i, 0}^{*}\right)=0$. Consider the families of local attractors and corresponding repellers defined in (4.1) and (4.2), and assume that the families of local unstable sets $\left\{W_{\eta, \rho}^{u}\left(\xi_{j}^{*}\right)(t)\right\}_{\eta \in[0,1]}$ behave lower semicontinuously.

Then, there exists $\eta_{0}>0$ such that for every $\eta \in\left[0, \eta_{0}\right]$ and every $j=1,2, \cdots, n$, the local atractor $A_{j, \eta}=\left\{A_{j, \eta}(t): t \in \mathbb{R}\right\}$ satisfies the conclusion of Lemma 2.12 for a neighborhood of $A_{j, \eta}$ in $X$ (see hypothesis (a) in Proposition 3.4).

Proof. Indeed, we fix $\delta_{1} \in(0, \varepsilon), \eta_{1}>0$ and $\delta_{1}^{\prime} \in\left(0, \delta_{1}\right)$ such that the conclusion of Lemma 4.10 holds. That is, for every $\eta \in\left[0, \eta_{1}\right]$ and each $j=1,2, \cdots, n$, we have

$$
T_{\eta}(t, s)\left(\mathcal{O}_{\delta_{1}^{\prime}}\left(A_{j, \eta}(s)\right)\right) \subset \mathcal{O}_{\delta_{1}}\left(A_{j, \eta}(t)\right) \text { whenever } t \geq s .
$$

On the other hand, from Lemma 4.4. there are $0<\delta_{3}^{\prime}<\delta_{2}^{\prime}<\delta_{1}^{\prime}$ and $\eta_{2} \in$ $\left(0, \eta_{1}\right]$ such that $\mathcal{O}_{\delta_{3}^{\prime}}\left(A_{j, \eta}(s)\right) \subset \mathcal{O}_{\delta_{2}^{\prime}}\left(A_{j, 0}\right) \subset \mathcal{O}_{\delta_{1}^{\prime}}\left(A_{j, \eta}(s)\right)$ for each $\eta \in\left[0, \eta_{2}\right]$, $j=1,2, \cdots, n$ and $s \in \mathbb{R}$. Hence, by (4.21), $\omega_{\eta}\left(\mathcal{O}_{\delta_{2}^{\prime}}\left(A_{j, 0}\right), s\right)^{1} \subset \mathcal{O}_{\delta_{1}}\left(A_{j, \eta}(s)\right)$ for all $s \in \mathbb{R}, \eta \in\left[0, \eta_{2}\right]$ and $j=1,2, \cdots, n$.

It is clear that $\omega_{\eta}\left(\mathcal{O}_{\delta_{2}^{\prime}}\left(A_{j, 0}\right), s\right)$ is contained in $A_{j, \eta}(s)$ for all $s \in \mathbb{R}$. Otherwise, there would be a global solution $\xi: \mathbb{R} \rightarrow X$ with $\xi(t) \in \mathcal{O}_{\delta_{1}}\left(A_{j, \eta}(t)\right)$ for all $t \in \mathbb{R}$

\footnotetext{
${ }^{1} \omega_{\eta}\left(\mathcal{O}_{\delta_{2}^{\prime}}\left(A_{j, 0}\right), s\right)$ indicates the $\omega$-limit set of $\mathcal{O}_{\delta_{2}^{\prime}}\left(A_{j, 0}\right)$ at time $s$ in the pullback sense with respect to the process $\left\{T_{\eta}(t, s): t \geq s\right\}$. We have that the set $\omega_{\eta}\left(\mathcal{O}_{\delta_{2}^{\prime}}\left(A_{j, 0}\right), s\right)$ pullback attracts $\mathcal{O}_{\delta_{2}^{\prime}}\left(A_{j, 0}\right)$ at time $s$ (see [4]).
} 
and such that $\xi\left(t_{0}\right) \notin A_{j, \eta}\left(t_{0}\right)$ for some $t_{0} \in \mathbb{R}$, but that contradicts the result of Proposition 4.7

Now the $\omega_{\eta}\left(\mathcal{O}_{\delta_{2}^{\prime}}\left(A_{j, 0}\right), t\right)$ pullback attracts $\mathcal{O}_{\delta_{2}^{\prime}}\left(A_{j, 0}\right)$ and, given $\delta>0$ and $t \in \mathbb{R}$, there exists $\tau_{t}>0$ such that

$$
T_{\eta}(t, t-\tau) \mathcal{O}_{\delta_{3}^{\prime}}\left(A_{j, \eta}(t-\tau)\right) \subset \mathcal{O}_{\delta}\left(A_{j, \eta}(t)\right)
$$

for all $\tau \geq \tau_{t}$.

From the continuity of the evolution process, choose $\delta^{\prime} \in\left(0, \min \left\{\delta, \delta_{3}^{\prime}\right\}\right)$ such that

$$
T_{\eta}(t, t-\tau) \mathcal{O}_{\delta^{\prime}}\left(A_{j, \eta}(t-\tau)\right) \subset \mathcal{O}_{\delta}\left(A_{j, \eta}(t)\right)
$$

for all $\tau \in\left[0, \tau_{t}\right]$.

This completes the proof of the proposition.

We now conclude that the class of a non-autonomous perturbation of a gradientlike semigroup satisfies all the hypotheses that we have used to develop the abstract framework in the previous section. We can therefore state the following result:

Theorem 4.12. Let $X$ be a metric space and, for each $\eta \in[0,1],\left\{T_{\eta}(t, s): t \geq s\right\}$ an evolution process on $X$ with a pullback attractor $\left\{\mathscr{A}_{\eta}(t): t \in \mathbb{R}\right\}$ which satisfies conditions $(a)-(e)$ in Theorem 4.1. Assume that $\mathcal{E}_{0}=\left\{y_{1,0}^{*}, \cdots, y_{n, 0}^{*}\right\}$ is reordered so that it is a Morse-decomposition for $\left\{T_{0}(t, 0): t \geq 0\right\}$ and that $\mathcal{E}_{\eta}$ is reordered in such a way that $\lim _{\eta \rightarrow 0+} \sup _{1 \leq i \leq n} \sup _{t \in \mathbb{R}} \mathbf{d}\left(\xi_{i, \eta}^{*}(t), y_{i, 0}^{*}\right)=0$. Then, there exists $\eta_{0}>0$ such that for all $\eta \in\left[0, \eta_{0}\right]$ the $n$-tuple $\left(\xi_{1, \eta}^{*}, \cdots, \xi_{n, \eta}^{*}\right)$ determines a Morse-decomposition for the pullback attractor of $\left\{T_{\eta}(t, s): t \geq s\right\}$. Furthermore, $\left\{T_{\eta}(t, s): t \geq s\right\}$ is a gradient evolution process with respect to the set of isolated global solutions, $\mathcal{E}_{\eta}=\left\{\xi_{1, \eta}^{*}, \cdots, \xi_{n, \eta}^{*}\right\}$, in the sense of Definition 3.1, with Lyapunov function $V_{\eta}$ : $\mathbb{R} \times X \rightarrow \mathbb{R}$ continuous in both variables and satisfying $V_{\eta}\left(t, \xi_{i, \eta}^{*}(t)\right)=i-1$, for all $t \in \mathbb{R}$ and $i=1, \cdots, n$.

4.1. Convergence of the Lyapunov functions. Now, following [2, we are going to study the continuity of the Lyapunov functions $V_{\eta}$ as $\eta$ tends to zero.

Theorem 4.13. Let $\left\{T_{\eta}(t, s): t \geq s\right\}_{\eta \in[0,1]}$ be a family of evolution processes in a metric space $X$ with a corresponding family of pullback attractors $\left\{\mathscr{A}_{\eta}\right\}_{\eta \in[0,1]}:=$ $\left\{\mathscr{A}_{\eta}(t): t \in \mathbb{R}\right\}_{\eta \in[0,1]}$ which satisfies conditions $(a)$ and $(d)$ in Theorem 4.1. Assume that $T_{0}(t, s)=S(t-s)$ for all $t \geq s$, where $\{S(t): t \geq 0\}$ is a semigroup.

For each $\eta \in[0,1]$, let $\left\{A_{\eta}\right\}:=\left\{A_{\eta}(t): t \in \mathbb{R}\right\}$ be a local attractor for $\left\{T_{\eta}(t, s)\right.$ : $t \geq s\}$ with associated repeller $\left\{A_{\eta}^{*}\right\}:=\left\{A_{\eta}^{*}(t): t \in \mathbb{R}\right\}$ and such that $A_{0}(t)=A_{0}$, where $A_{0}$ is a local attractor for $\left\{T_{0}(t, 0)=S(t): t \geq 0\right\}$.

Suppose that $\left\{A_{\eta}\right\}_{\eta \in[0,1]},\left\{A_{\eta}^{*}\right\}_{\eta \in[0,1]}$, and $\left\{\mathscr{A}_{\eta}\right\}_{\eta \in[0,1]}$ are continuous at $\eta=0$; that is,

$$
\begin{aligned}
& \lim _{\eta \rightarrow 0^{+}} \sup _{t \in \mathbb{R}} \mathbf{d}_{H}\left(A_{\eta}(t), A_{0}\right)=0, \\
& \lim _{\eta \rightarrow 0^{+}} \sup _{t \in \mathbb{R}} \mathbf{d}_{H}\left(A_{\eta}^{*}(t), A_{0}^{*}\right)=0, \\
& \lim _{\eta \rightarrow 0^{+}} \sup _{t \in \mathbb{R}} \mathbf{d}_{H}\left(\mathscr{A}_{\eta}(t), \mathscr{A}_{0}\right)=0 .
\end{aligned}
$$

Finally, for each $\eta \in[0,1]$, let $f_{\eta}: \mathbb{R} \times X \rightarrow \mathbb{R}$ be the Lyapunov function associated to the attractor repeller pair $\left(A_{\eta}, A_{\eta}^{*}\right)$ defined by

$$
f_{\eta}(t, z):=k_{\eta}(t, z)+h_{\eta}(t, z),(t, z) \in \mathbb{R} \times X,
$$


where

$$
\begin{gathered}
h_{\eta}(t, z):=\sup _{r \geq 0} \operatorname{dist}\left(T_{\eta}(r+t, t) z, \mathscr{A}_{\eta}(t+r)\right),(t, z) \in \mathbb{R} \times X, \\
k_{\eta}(t, z):=\sup _{r \geq 0} \frac{\operatorname{dist}\left(z, A_{\eta}(t)\right)}{\operatorname{dist}\left(z, A_{\eta}(t)\right)+\operatorname{dist}\left(z, A_{\eta}^{*}(t)\right)},(t, z) \in \mathbb{R} \times X .
\end{gathered}
$$

Then, for each compact subset $K$ of $X$ we have

$$
\lim _{\eta \rightarrow 0^{+}} \sup _{t \in \mathbb{R}} \sup _{z \in K}\left|f_{\eta}(t, z)-f_{0}(z)\right|=0 .
$$

Proof. We split the proof into three steps:

Step 1 . We have the following convergence:

$$
\lim _{\eta \rightarrow 0^{+}} \sup _{t \in \mathbb{R}} \sup _{z \in X}\left|l_{\eta}(t, z)-l_{0}(z)\right|=0 .
$$

Note that, for each $\eta \in[0,1], z \in X$ and $t \in \mathbb{R}$,

$$
\left|\operatorname{dist}\left(z, A_{\eta}(t)\right)-\operatorname{dist}\left(z, A_{0}\right)\right| \leq \mathbf{d}_{H}\left(A_{\eta}(t), A_{0}\right)
$$

and

$$
\left|\operatorname{dist}\left(z, A_{\eta}^{*}(t)\right)-\operatorname{dist}\left(z, A_{0}^{*}\right)\right| \leq \mathbf{d}_{H}\left(A_{\eta}^{*}(t), A_{0}^{*}\right) .
$$

Now, given $\eta \in[0,1], t \in \mathbb{R}$ and $z \in X$, we have

$$
\begin{aligned}
& l_{\eta}(z, t)-l_{0}(z) \\
& =\frac{\left[\operatorname{dist}\left(z, A_{\eta}(t)\right)-\operatorname{dist}\left(z, A_{0}\right)\right] \operatorname{dist}\left(z, A_{0}^{*}\right)+\operatorname{dist}\left(z, A_{0}\right)\left[\operatorname{dist}\left(z, A_{0}^{*}\right)-\operatorname{dist}\left(z, A_{\eta}^{*}(t)\right)\right]}{\left[\operatorname{dist}\left(z, A_{\eta}(t)\right)+\operatorname{dist}\left(z, A_{\eta}^{*}(t)\right)\right]\left[\operatorname{dist}\left(z, A_{0}\right)+\operatorname{dist}\left(z, A_{0}^{*}\right)\right]} .
\end{aligned}
$$

Since $\mathbf{d}\left(A_{0}, A_{0}^{*}\right)>\mu$ for some $\mu>0$, using the fact that the families of local attractors and their corresponding repellers are continuous, there exists $\tilde{\eta} \in(0,1]$ such that $\mathbf{d}\left(A_{\eta}(t), A_{\eta}^{*}(t)\right) \geq \mu$, for all $\eta \in[0, \tilde{\eta}]$. From (4.22) and (4.23)

$$
\begin{aligned}
\left|l_{\eta}(t, z)-l_{0}(z)\right| & \leq \frac{1}{\operatorname{dist}\left(z, A_{\eta}(t)\right)+\operatorname{dist}\left(z, A_{\eta}^{*}(t)\right)}\left[\mathbf{d}_{H}\left(A_{\eta}(t), A_{0}\right)+\mathbf{d}_{\mathrm{H}}\left(A_{\eta}^{*}(t), A_{0}^{*}\right)\right] \\
& \leq \frac{1}{\mu}\left[\mathbf{d}_{H}\left(A_{\eta}(t), A_{0}\right)+\mathbf{d}_{H}\left(A_{\eta}^{*}(t), A_{0}^{*}\right)\right],
\end{aligned}
$$

for each $z \in X$ and $\eta \in[0, \tilde{\eta}]$. Hence

$$
\left|l_{\eta}(t, z)-l_{0}(z)\right| \leq \frac{1}{\mu}\left[\mathbf{d}_{H}\left(A_{\eta}(t), A_{0}\right)+\mathbf{d}_{H}\left(A_{\eta}^{*}(t), A_{0}^{*}\right)\right],
$$

for all $t \in \mathbb{R}, z \in X$ and $\eta \in[0, \tilde{\eta}]$. From the continuity assumptions (on the local attractors and corresponding repellers) the uniform convergence (in $\mathbb{R} \times X$ ) of $l_{\eta}$ to $l_{0}$ follows.

Step 2. For every compact $K \subset X$ we have

$$
\lim _{\eta \rightarrow 0^{+}} \sup _{t \in \mathbb{R}} \sup _{z \in K}\left|k_{\eta}(t, z)-k_{0}(z)\right|=0 .
$$


Indeed, given $z \in X$ we know, by Lemma 2.13(iii) from [1], that $S(t) z \stackrel{t \rightarrow \infty}{\rightarrow}$ $A_{0} \cup A_{0}^{*}$. Then consider the following three cases:

Case 1: $S(t) z \stackrel{t \rightarrow \infty}{\rightarrow} A_{0}$ with $l_{0}(z)>0$. Choose $0<\theta<l_{0}(z)$. Since $l_{0}: X \rightarrow \mathbb{R}$ is continuous, there is a $\sigma_{1}>0$ such that $l_{0}\left(\mathcal{O}_{\sigma_{1}}(z)\right) \subset\left(\frac{\theta}{2}, 1\right]$. From Step 1 , there is an $\eta_{0} \in(0,1]$ such that $l_{\eta}\left(t, \mathcal{O}_{\sigma_{1}}(z)\right) \subset(\theta, 1]$ for all $\eta \in\left[0, \eta_{0}\right]$ and $t \in \mathbb{R}$.

Again using the continuity of $l_{0}: X \rightarrow \mathbb{R}$, given $0<\alpha<\frac{\theta}{2}$, there is a $\delta>0$ such that $l_{0}\left(\mathcal{O}_{\delta}\left(A_{0}\right)\right) \subset[0, \alpha)$. Now, from Lemma 4.10 there is a $\delta^{\prime} \in\left(0, \frac{\delta}{2}\right)$ and $\eta_{1} \in\left(0, \eta_{0}\right]$ such that

$$
T_{\eta}(t, s)\left(\mathcal{O}_{\delta^{\prime}}\left(A_{\eta}(s)\right)\right) \subset \mathcal{O}_{\frac{\delta}{2}}\left(A_{\eta}(t)\right) \text { whenever } t \geq s, \forall \eta \in\left[0, \eta_{1}\right] .
$$
that

From the lower semicontinuity of $\left\{A_{\eta}\right\}_{\eta \in[0,1]}$ at $\eta=0$, there is $\eta_{2} \in\left(0, \eta_{1}\right]$ such

$$
A_{0} \subset \mathcal{O}_{\frac{\delta^{\prime}}{2}}\left(A_{\eta}(t)\right) \text { for all } t \in \mathbb{R} \forall \eta \in\left[0, \eta_{2}\right] .
$$

From the fact that $T_{0}(t) z \stackrel{t \rightarrow \infty}{\rightarrow} A_{0}$, choose $t_{0}>0$ such that $T_{0}\left(t_{0}\right) z \in \mathcal{O}_{\frac{\delta^{\prime}}{4}}\left(A_{0}\right)$, and from the continuity of $T_{0}\left(t_{0}\right): X \rightarrow X$ choose $\sigma_{2} \in\left(0, \sigma_{1}\right]$ such that $T_{0}\left(t_{0}\right)\left(\mathcal{O}_{\sigma_{2}}(z)\right)$ $\subset \mathcal{O}_{\frac{\delta^{\prime}}{4}}\left(A_{0}\right)$. From hypothesis $(d)$, we can find $\sigma_{3} \in\left(0, \sigma_{2}\right]$ and $\eta_{3} \in\left(0, \eta_{2}\right]$ such that for all $\eta \in\left[0, \eta_{3}\right]$ we have $T_{\eta}\left(t_{0}+s, s\right)\left(\mathcal{O}_{\sigma_{3}}(z)\right) \subset \mathcal{O}_{\frac{\delta^{\prime}}{2}}\left(A_{0}\right)$ for all $s \in \mathbb{R}$. From this and (4.25), we obtain that $T_{\eta}\left(t_{0}+s, s\right)\left(\mathcal{O}_{\sigma_{3}}(z)\right) \subset \mathcal{O}_{\delta^{\prime}}\left(A_{\eta}(t)\right)$ for all $s \in \mathbb{R}$ and $t \in \mathbb{R}$ when $\eta \in\left[0, \eta_{3}\right]$, and from (4.24), in particular, we conclude that

$$
T_{\eta}(t+s, s)\left(\mathcal{O}_{\sigma_{3}}(z)\right) \subset \mathcal{O}_{\frac{\delta}{2}}\left(A_{\eta}(t+s)\right) \text { for all } t \geq t_{0}, \eta \in\left[0, \eta_{3}\right] \text { and } s \in \mathbb{R} \text {. }
$$

On the other hand, observe that, from the uniform convergence of $l_{\eta} \stackrel{\eta \rightarrow 0^{+}}{\rightarrow} l_{0}$ in $\mathbb{R} \times X$, we obtain $\eta_{4} \in\left(0, \eta_{3}\right]$ so that, for each $\eta \in\left[0, \eta_{4}\right]$, it holds that $l_{\eta}\left(t, \mathcal{O}_{\delta}\left(A_{0}\right)\right) \subset$ $[0,2 \alpha)$ for all $t \in \mathbb{R}$. From the upper semicontinuity of $\left\{A_{\eta}\right\}_{\eta \in[0,1]}$ at $\eta=0$, there exists $\eta_{5} \in\left(0, \eta_{4}\right]$ such that, if $\eta \in\left[0, \eta_{5}\right]$, then $A_{\eta}(t) \subset \mathcal{O}_{\frac{\delta}{2}}\left(A_{0}\right)$ for all $t \in \mathbb{R}$. Therefore, $\mathcal{O}_{\frac{\delta}{2}}\left(A_{\eta}(t)\right) \subset \mathcal{O}_{\delta}\left(A_{0}\right)$ for all $\eta \in\left[0, \eta_{5}\right]$ and $t \in \mathbb{R}$. So $l_{\eta}\left(t, \mathcal{O}_{\frac{\delta}{2}}\left(A_{\eta}(\tau)\right)\right) \subset$ $[0,2 \alpha)$ for all $\eta \in\left[0, \eta_{5}\right]$ and $t, \tau$ in $\mathbb{R}$. From (4.26), we have that

$$
\sup _{t \geq t_{0}} l_{\eta}\left(t+s, T_{\eta}(t+s, s) w\right) \leq 2 \alpha<\theta<l_{\eta}(s, w) \leq k_{\eta}(s, w),
$$

for each $\eta \in\left[0, \eta_{5}\right], s \in \mathbb{R}$ and $w \in \mathcal{O}_{\sigma_{3}}(z) \subset \mathcal{O}_{\sigma_{1}}(z)$. Consequently $k_{\eta}(s, w)=$ $\sup l_{\eta}\left(t+s, T_{\eta}(t+s, s) w\right)$ for all $\eta \in\left[0, \eta_{5}\right], s \in \mathbb{R}$ and $w \in \mathcal{O}_{\sigma_{3}}(z)$.

Finally, given $\varepsilon>0$, from Step 1 , there exists $\eta_{6} \in\left(0, \eta_{5}\right]$ such that

$$
\left|l_{\eta}(t, x)-l_{0}(x)\right|<\frac{\varepsilon}{2} \text { for all } \eta \in\left[0, \eta_{6}\right], x \in X \text { and } t \in \mathbb{R} .
$$

From the uniform continuity of the function $l_{0}: X \rightarrow \mathbb{R}$, consider $\beta>0$ such that if $x, x^{\prime} \in X$ satisfy $\mathbf{d}\left(x, x^{\prime}\right)<\beta$, then $\left|l_{0}(x)-l_{0}\left(x^{\prime}\right)\right|<\frac{\varepsilon}{2}$ so that, by the convergence in $(d)$, we can choose $\eta_{7} \in\left(0, \eta_{6}\right]$ and $\sigma_{4} \in\left(0, \sigma_{3}\right]$ such that

$$
\sup _{s \in \mathbb{R}} \sup _{w \in \mathcal{O}_{\sigma_{4}}(z)} \sup _{0 \leq t \leq t_{0}} \mathbf{d}\left(T_{\eta}(t+s, s) w, S(t) w\right)<\beta \text { for all } \eta \in\left[0, \eta_{7}\right] .
$$

Thus

$$
\begin{aligned}
\left|l_{\eta}\left(t+s, T_{\eta}(t+s, s) w\right)-l_{0}(S(t) w)\right| \leq & \left|l_{\eta}\left(t+s, T_{\eta}(t+s, s) w\right)-l_{0}\left(T_{\eta}(t+s, s) w\right)\right| \\
& +\left|l_{0}\left(T_{\eta}(t+s, s) w\right)-l_{0}\left(T_{0}(t) w\right)\right|<\varepsilon,
\end{aligned}
$$


for all $w \in \mathcal{O}_{\sigma_{4}}(z), t \in\left[0, t_{0}\right], s \in \mathbb{R}$ and $\eta \in\left[0, \eta_{7}\right]$. This implies that

$$
\sup _{s \in \mathbb{R}} \sup _{w \in \mathcal{O}_{\sigma_{4}}(z)}\left|k_{\eta}(s, w)-k_{0}(w)\right| \leq \varepsilon, \text { for all } \eta \in\left[0, \eta_{7}\right]
$$

where $\sigma_{4}>0$ and $\eta_{7}$ only depends on $z \in X$ and $\varepsilon>0$.

Case 2: $l_{0}(z)=0$. Under these conditions, note that $z \in A_{0}$ and, consequently, $k_{0}(z)=0$.

Given $\varepsilon>0$, by the continuity of $l_{0}: X \rightarrow \mathbb{R}$, take $\delta>0$ such that $l_{0}\left(\mathcal{O}_{\delta}\left(A_{0}\right)\right) \subset$ $\left[0, \frac{\varepsilon}{4}\right)$.

Now, the uniform convergence of $\left(l_{\eta}\right)_{\eta \in[0,1]}$ to $l_{0}$ in $\mathbb{R} \times X$ implies the existence of $\eta_{0} \in(0,1]$ such that

$$
l_{\eta}\left(t, \mathcal{O}_{\delta}\left(A_{0}\right)\right) \subset\left[0, \frac{\varepsilon}{2}\right) \text { for each } \eta \in\left[0, \eta_{0}\right] \text { and each } t \in \mathbb{R} .
$$

By the upper semicontinuity of $\left\{A_{\eta}\right\}_{\eta \in[0,1]}$ at $\eta=0$, we have the existence of $\eta_{1} \in\left(0, \eta_{0}\right]$ such that for all $\eta \in\left[0, \eta_{1}\right]$ and all $t \in \mathbb{R}$ we have $A_{\eta}(t) \subset \mathcal{O}_{\frac{\delta}{2}}\left(A_{0}\right)$, from which $\mathcal{O}_{\frac{\delta}{2}}\left(A_{\eta}(t)\right) \subset \mathcal{O}_{\delta}\left(A_{0}\right)$ if $\eta \in\left[0, \eta_{1}\right]$ and $t \in \mathbb{R}$. From (4.28) we conclude that for all $\eta \in\left[0, \eta_{1}\right]$,

$$
l_{\eta}\left(t, \mathcal{O}_{\frac{\delta}{2}}\left(A_{\eta}(s)\right)\right) \subset\left[0, \frac{\varepsilon}{2}\right) \text { for all } s, t \in \mathbb{R} .
$$

Choose $\eta_{2} \in\left(0, \eta_{1}\right]$ and $\delta^{\prime} \in\left(0, \frac{\delta}{2}\right)$, by Lemma 4.10 such that

$$
T_{\eta}(t, s)\left(\mathcal{O}_{\delta^{\prime}}\left(A_{\eta}(s)\right)\right) \subset \mathcal{O}_{\frac{\delta}{2}}\left(A_{\eta}(t)\right) \text { whenever } t \geq s \text { and for all } \eta \in\left[0, \eta_{2}\right] .
$$

Finally, from the lower semicontinuity of $\left\{A_{\eta}\right\}_{\eta \in[0,1]}$ at $\eta=0$, there is a $\eta_{3} \in$ $\left(0, \eta_{2}\right]$ such that

$$
A_{0} \subset \mathcal{O}_{\frac{\delta^{\prime}}{2}}\left(A_{\eta}(t)\right) \text { for all } \eta \in\left[0, \eta_{3}\right] \text { and } t \in \mathbb{R} .
$$

Thus, from (4.31) and (4.30), for $\eta \in\left[0, \eta_{3}\right]$, for $s \in \mathbb{R}$ and $w \in \mathcal{O}_{\frac{\delta^{\prime}}{2}}\left(A_{0}\right) \subset$ $\mathcal{O}_{\delta^{\prime}}\left(A_{\eta}(s)\right)$, we have that $T_{\eta}(t+s, s) w \in \mathcal{O}_{\frac{\delta}{2}}\left(A_{\eta}(t+s)\right)$ for all $t \geq 0$. From (4.29) it follows that

$$
k_{\eta}(s, w)=\sup _{t \geq 0} l_{\eta}\left(t+s, T_{\eta}(t+s, s) w\right) \leq \frac{\varepsilon}{2},
$$

for all $\eta \in\left[0, \eta_{3}\right], s \in \mathbb{R}$ and $w \in \mathcal{O}_{\frac{\delta^{\prime}}{2}}\left(A_{0}\right)$. In particular,

$$
\sup _{s \in \mathbb{R}} \sup _{w \in \mathcal{O}_{\frac{\delta^{\prime}}{2}}\left(A_{0}\right)}\left|k_{\eta}(s, w)-k_{0}(w)\right| \leq \varepsilon, \text { for all } \eta \in\left[0, \eta_{3}\right],
$$

where $\delta^{\prime}>0$ and $\eta_{3}$ only depend on $\varepsilon>0$ and $A_{0}$.

Case 3: $T_{0}(t) z \stackrel{t \rightarrow \infty}{\rightarrow} A_{0}^{*}$. In this case $k_{0}(z)=1$. By the continuity of $l_{0}: X \rightarrow \mathbb{R}$, given $\varepsilon>0$, let $\delta>0$ such that

$$
l_{0}\left(\mathcal{O}_{\delta}\left(A_{0}^{*}\right)\right) \subset\left(1-\frac{\varepsilon}{4}, 1\right]
$$

and, by the uniform convergence $l_{\eta} \stackrel{\eta \rightarrow 0^{+}}{\rightarrow} l_{0}$ in $\mathbb{R} \times X$, take $\eta_{0} \in(0,1]$ such that

$$
l_{\eta}\left(t, \mathcal{O}_{\delta}\left(A_{0}^{*}\right)\right) \subset\left(1-\frac{\varepsilon}{2}, 1\right] \text { for all } \eta \in\left[0, \eta_{0}\right] \text { and } t \in \mathbb{R} .
$$

On the other hand, consider $t_{0}>0$ such that $T_{0}\left(t_{0}\right) z \in \mathcal{O}_{\frac{\delta}{2}}\left(A_{0}^{*}\right)$ and, from the continuity of $T_{0}\left(t_{0}\right): X \rightarrow X$, take $\sigma_{1}>0$ such that $T_{0}\left(t_{0}\right)\left(\mathcal{O}_{\sigma_{1}}(z)\right) \subset \mathcal{O}_{\frac{\delta}{2}}\left(A_{0}^{*}\right)$. By 
hypothesis $(d)$, let $\eta_{1} \in\left(0, \eta_{0}\right]$ and $\sigma_{2} \in\left(0, \sigma_{1}\right]$ such that $T_{\eta}\left(t_{0}+s, s\right)\left(\mathcal{O}_{\sigma_{2}}(z)\right) \subset$ $\mathcal{O}_{\delta}\left(A_{0}^{*}\right)$ for all $\eta \in\left[0, \eta_{1}\right]$ and all $s \in \mathbb{R}$.

Finally, from (4.33) we deduce that $l_{\eta}\left(t_{0}+s, T_{\eta}\left(t_{0}+s, s\right)\left(\mathcal{O}_{\sigma_{2}}(z)\right)\right) \subset\left(1-\frac{\varepsilon}{2}, 1\right]$ for all $\eta \in\left[0, \eta_{1}\right]$ and all $s \in \mathbb{R}$. Thus, $1-\frac{\varepsilon}{2}<l_{\eta}\left(t_{0}+s, T_{\eta}\left(t_{0}+s, s\right) w\right) \leq k_{\eta}(s, w) \leq 1$ for all $w \in \mathcal{O}_{\sigma_{2}}(z), s \in \mathbb{R}$ and $\eta \in\left[0, \eta_{1}\right]$. This implies that $\left|k_{\eta}(s, w)-k_{0}(w)\right| \leq \varepsilon$ for $\eta \in\left[0, \eta_{1}\right], s \in \mathbb{R}$ and $w \in \mathcal{O}_{\sigma_{2}}(z)$ and

$$
\sup _{s \in \mathbb{R}} \sup _{w \in \mathcal{O}_{\sigma_{2}}(z)}\left|k_{\eta}(s, w)-k_{0}(w)\right| \leq \varepsilon, \text { for } \eta \in\left[0, \eta_{1}\right],
$$

where $\sigma_{2}>0$ and $\eta_{1}$ only depends on $A_{0}^{*}$ and $\varepsilon>0$.

Now, joining Cases 1, 2 and 3, we have that:

Given a compact subset $K \subset X$ and $\varepsilon>0$, by (4.27), (4.32) and (4.34), there exist an open subset $U=U(\varepsilon, K) \subset X$ with $K \subset U$, and an index $\eta^{\prime}=\eta^{\prime}(\varepsilon, K)>0$ such that

$$
\sup _{s \in \mathbb{R}} \sup _{w \in U}\left|k_{\eta}(s, w)-k_{0}(w)\right| \leq \varepsilon, \text { for all } \eta \in\left[0, \eta^{\prime}\right]
$$

from which we can conclude that $\lim _{\eta \rightarrow 0^{+}} \sup _{s \in \mathbb{R} w \in K}\left|k_{\eta}(s, w)-k_{0}(w)\right|=0$.

Step 3. For every compact $K \subset X$ we have

$$
\lim _{\eta \rightarrow 0^{+}} \sup _{t \in \mathbb{R}} \sup _{z \in K}\left|h_{\eta}(t, z)-h_{0}(z)\right|=0 .
$$

Indeed, given $z \in X$ now consider two cases:

Case 1: $\operatorname{dist}\left(z, \mathscr{A}_{0}\right)>0$. Given $\alpha>0$ with $0<\alpha<\operatorname{dist}\left(z, \mathscr{A}_{0}\right)$, let, by Lemma 4.10, $\alpha^{\prime} \in(0, \alpha)$ and $\eta_{0} \in(0,1]$ such that

$$
T_{\eta}(t, s)\left(\mathcal{O}_{\alpha^{\prime}}\left(\mathscr{A}_{\eta}(s)\right)\right) \subset \mathcal{O}_{\alpha}\left(\mathscr{A}_{\eta}(t)\right) \text { whenever } t \geq s, \text { for all } \eta \in\left[0, \eta_{0}\right] .
$$

Choose $t_{0}>0$ such that $T_{0}\left(t_{0}\right) z \in \mathcal{O}_{\frac{\alpha^{\prime}}{4}}\left(\mathscr{A}_{0}\right)$ and, by the continuity of $T_{0}\left(t_{0}\right)$ : $X \rightarrow X$, let $\sigma_{1}>0$ such that $T_{0}\left(t_{0}\right)\left(\mathcal{O}_{\sigma_{1}}(z)\right) \subset \mathcal{O}_{\frac{\alpha^{\prime}}{4}}\left(\mathscr{A}_{0}\right)$.

Now, from $(d)$, let $\eta_{1} \in\left(0, \eta_{0}\right]$ and $\sigma_{2} \in\left(0, \sigma_{1}\right]$ such that $T_{\eta}\left(t_{0}+s, s\right)\left(\mathcal{O}_{\sigma_{2}}(z)\right) \subset$ $\mathcal{O}_{\frac{\alpha^{\prime}}{2}}\left(\mathscr{A}_{0}\right)$ for each $\eta \in\left[0, \eta_{1}\right]$ and each $s \in \mathbb{R}$. From the lower semicontinuity of ${ }^{2}\left\{\mathscr{A}_{\eta}\right\}_{\eta \in[0,1]}$ at $\eta=0$, choose $\eta_{2} \in\left(0, \eta_{1}\right]$ such that $\mathscr{A}_{0} \subset \mathcal{O}_{\frac{\alpha^{\prime}}{2}}\left(\mathscr{A}_{\eta}(t)\right)$ for all $\eta \in\left[0, \eta_{2}\right]$ and $t \in \mathbb{R}$. Hence, $\mathcal{O}_{\frac{\alpha^{\prime}}{2}}\left(\mathscr{A}_{0}\right) \subset \mathcal{O}_{\alpha^{\prime}}\left(\mathscr{A}_{\eta}(t)\right)$ for $\eta \in\left[0, \eta_{2}\right]$ and $t \in \mathbb{R}$. In particular, $T_{\eta}\left(t_{0}+s, s\right)\left(\mathcal{O}_{\sigma_{2}}(z)\right)^{2} \subset \mathcal{O}_{\alpha^{\prime}}\left(\mathscr{A}_{\eta}\left(t_{0}+s\right)\right)$ for all $\eta \in\left[0, \eta_{2}\right]$ and $s \in \mathbb{R}$. From (4.35) we obtain that $T_{\eta}(t+s, s)\left(\mathcal{O}_{\sigma_{2}}(z)\right) \subset \mathcal{O}_{\alpha}\left(\mathscr{A}_{\eta}(t+s)\right)$ for all $\eta \in\left[0, \eta_{2}\right]$, all $s \in \mathbb{R}$ and $t \geq t_{0}$. Consequently,

(4.36) $\sup _{t \geq t_{0}} \operatorname{dist}\left(T_{\eta}(t+s, s) w, \mathscr{A}_{\eta}(t+s)\right) \leq \alpha$ for all $\eta \in\left[0, \eta_{2}\right], s \in \mathbb{R}, w \in \mathcal{O}_{\sigma_{2}}(z)$.

On the other hand, for all $w \in X$, all $t \in \mathbb{R}$ and all $\eta \in[0,1]$, we have

$$
\left|\operatorname{dist}\left(w, \mathscr{A}_{\eta}(t)\right)-\operatorname{dist}\left(w, \mathscr{A}_{0}\right)\right| \leq \mathbf{d}_{H}\left(\mathscr{A}_{\eta}(t), \mathscr{A}_{0}\right) .
$$

Then, we can choose $\eta_{3} \in\left(0, \eta_{2}\right]$ and $\sigma_{3} \in\left(0, \sigma_{2}\right]$ such that $\operatorname{dist}\left(w, \mathscr{A}_{\eta}(t)\right)>\alpha$ for all $\eta \in\left[0, \eta_{3}\right], t \in \mathbb{R}$ and $w \in \mathcal{O}_{\sigma_{3}}(z)$. This and (4.36) implies that

$$
\sup _{t \geq t_{0}} \operatorname{dist}\left(T_{\eta}(t+s, s) w, \mathscr{A}_{\eta}(t+s)\right) \leq \alpha<\operatorname{dist}\left(w, \mathscr{A}_{\eta}(s)\right)
$$


for all $\eta \in\left[0, \eta_{3}\right], s \in \mathbb{R}$ and $w \in \mathcal{O}_{\sigma_{3}}(z)$. So,

$$
h_{\eta}(s, w)=\sup _{0 \leq t \leq t_{0}} \operatorname{dist}\left(T_{\eta}(t+s, s) w, \mathscr{A}_{\eta}(t+s)\right)
$$

for $\eta \in\left[0, \eta_{3}\right], s \in \mathbb{R}$ and $w \in \mathcal{O}_{\sigma_{3}}(z)$.

Note that, for all $w \in X, \eta \in[0,1], s \in \mathbb{R}$ and $t \geq 0$, we have that

$$
\begin{aligned}
\left|\operatorname{dist}\left(T_{\eta}(t+s, s) w, \mathscr{A}_{\eta}(t+s)\right)-\operatorname{dist}\left(S(t) w, \mathscr{A}_{0}\right)\right| & \leq \mathbf{d}_{H}\left(\mathscr{A}_{\eta}(t+s), \mathscr{A}_{0}\right) \\
& +\mathbf{d}\left(T_{\eta}(t+s, s) w, S(t) w\right),
\end{aligned}
$$

so that, for all $\eta \in\left[0, \eta_{3}\right]$,

$$
\begin{aligned}
\sup _{s \in \mathbb{R}} \sup _{w \in \mathcal{O}_{\sigma_{3}}(z)}\left|h_{\eta}(s, w)-h_{0}(w)\right| & \leq \sup _{s \in \mathbb{R}} \mathbf{d}_{H}\left(\mathscr{A}_{\eta}(s), \mathscr{A}_{0}\right) \\
& +\sup _{s \in \mathbb{R}} \sup _{w \in \mathcal{O}_{\sigma_{3}}(z)} \sup _{0 \leq t \leq t_{0}} \mathbf{d}\left(T_{\eta}(t+s, s) w, S(t) w\right),
\end{aligned}
$$

and so, it is easy to see that, given $\varepsilon>0$, there exist $\sigma \in\left(0, \sigma_{3}\right]$ and $\eta_{4} \in\left(0, \eta_{3}\right]$ such that

$$
\sup _{s \in \mathbb{R}} \sup _{w \in \mathcal{O}_{\sigma}(z)}\left|h_{\eta}(s, w)-h_{0}(w)\right| \leq \varepsilon \text { for all } \eta \in\left[0, \eta_{4}\right] .
$$

Case 2: $\operatorname{dist}\left(z, \mathscr{A}_{0}\right)=0 ;$ that is, $z \in \mathscr{A}_{0}$. From Lemma 4.10, given $\varepsilon>0$, there are $\varepsilon^{\prime} \in\left(0, \frac{\varepsilon}{2}\right)$ and $\eta_{0} \in(0,1]$ such that

$$
T_{\eta}(t, s)\left(\mathcal{O}_{\varepsilon^{\prime}}\left(\mathscr{A}_{\eta}(s)\right)\right) \subset \mathcal{O}_{\frac{\varepsilon}{2}}\left(\mathscr{A}_{\eta}(t)\right) \text { for each } t \geq s \text { and } \eta \in\left[0, \eta_{0}\right] .
$$

Also, since $\left\{\mathscr{A}_{\eta}\right\}_{\eta \in[0,1]}$ is lower semicontinuous at $\eta=0$, we can choose $\eta_{1} \in$ $\left(0, \eta_{0}\right]$ such that $\mathscr{A}_{0} \subset \mathcal{O}_{\frac{\varepsilon^{\prime}}{2}}\left(\mathscr{A}_{\eta}(s)\right)$ whenever $\eta \in\left[0, \eta_{1}\right]$ and $s \in \mathbb{R}$. It follows that $\mathcal{O}_{\frac{\varepsilon^{\prime}}{2}}\left(\mathscr{A}_{0}\right) \subset \mathcal{O}_{\varepsilon^{\prime}}\left(\mathscr{A}_{\eta}(s)\right)$ for all $\eta \in\left[0, \eta_{1}\right]$ and $s \in \mathbb{R}$. From this and (4.38) we have that

$$
T_{\eta}(t, s)\left(\mathcal{O}_{\frac{\varepsilon^{\prime}}{2}}\left(\mathscr{A}_{0}\right)\right) \subset \mathcal{O}_{\frac{\varepsilon}{2}}\left(\mathscr{A}_{\eta}(t)\right) \text { if } \eta \in\left[0, \eta_{1}\right] \text { and } t \geq s .
$$

Consequently, $h_{\eta}(s, w)=\sup _{t \geq 0} \operatorname{dist}\left(T_{\eta}(t+s, s) w, \mathscr{A}_{\eta}(t+s)\right) \leq \frac{\varepsilon}{2}$ for all $\eta \in\left[0, \eta_{1}\right]$ and $w \in \mathcal{O}_{\frac{\varepsilon^{\prime}}{2}}\left(\mathscr{A}_{0}\right)$, so that

$$
\sup _{s \in \mathbb{R}} \sup _{w \in \mathcal{O}_{\frac{\varepsilon^{\prime}}{2}}\left(\mathscr{A}_{0}\right)}\left|h_{\eta}(s, w)-h_{0}(w)\right| \leq \varepsilon \text { for all } \eta \in\left[0, \eta_{1}\right] .
$$

In these conditions, given $\varepsilon>0$ and $z \in X$, there are $\sigma=\sigma(\varepsilon, z)>0$ and $\eta^{\prime}=\eta^{\prime}(\varepsilon, z)>0$ such that

$$
\sup _{s \in \mathbb{R}} \sup _{w \in \mathcal{O}_{\sigma}(z)}\left|h_{\eta}(s, w)-h_{0}(w)\right| \leq \varepsilon \text { for all } \eta \in\left[0, \eta^{\prime}\right] .
$$

We now obtain the convergence $\lim _{\eta \rightarrow 0^{+}} \sup _{t \in \mathbb{R}} \sup _{z \in K}\left|h_{\eta}(t, z)-h_{0}(z)\right|=0$ by an argument similar to that of Step 2. This completes the proof.

\section{Applications}

In this section we consider some applications of the abstract results in the previous sections and conclude that some non-autonomous evolutions processes possess a Morse-decomposition and, therefore, a Lyapunov function. 
Example 5.1. Consider the initial boundary value problem

$$
\left\{\begin{array}{l}
u_{t}=u_{x x}+\lambda f_{\eta}(t, u), x \in(0, \pi), t>\tau, \\
u_{x}(0, t)=u_{x}(\pi, t)=0, t>\tau, \\
u(\cdot, \tau)=\phi_{0} \in H_{0}^{1}(0, \pi),
\end{array}\right.
$$

where $\lambda \in[0, \infty), \eta \in[0,1]$ and $f_{\eta} \in C^{2}\left(\mathbb{R}^{2}, \mathbb{R}\right)$ satisfying:

1) There exists $M>0$ such that $f_{\eta}: \mathbb{R}^{2} \rightarrow \mathbb{R}$ is such that $f_{\eta}(t, u) u<0$ for all $(t, u) \in \mathbb{R}^{2}$ with $|u|>M$. Suppose also that $f_{0}(t, u)=f_{0}(u)$ for all $t \in \mathbb{R}$.

2) For each $r>0$,

$\lim _{\eta \rightarrow 0+} \sup _{t \in \mathbb{R}} \sup _{|u| \leq r}\left\{\left|f_{\eta}(t, u)-f_{0}(u)\right|+\left|\left(f_{\eta}\right)_{u}(t, u)-f_{0}^{\prime}(u)\right|+\left|\left(f_{\eta}\right)_{u u}(t, u)-f_{0}^{\prime \prime}(u)\right|\right\}=0$.

It is not difficult to see that, under these assumptions, problem (5.1) possesses a pullback attractor $\left\{\mathscr{A}_{\eta}(t): t \in \mathbb{R}\right\}$.

If (5.1) with $\eta=0$ has a finite number of equilibria, all of them hyperbolic, the hypotheses of Theorem 4.12 are clearly satisfied and there exists $\eta_{0}>0$ such that $\left\{\mathscr{A}_{\eta}(t): t \in \mathbb{R}\right\}$ has a Morse-decomposition and a continuous (uniformly in $\mathbb{R} \times K$ for each compact subset $K$ of $\left.H_{0}^{1}(0, \pi)\right)$ Lyapunov function $V_{\eta}: \mathbb{R} \times H_{0}^{1}(0, \pi) \rightarrow \mathbb{R}$ (in the sense of Definition 3.1).

Example 5.2. Consider a general cascade system of the form

$$
\left\{\begin{array}{ccc}
\dot{u}_{1} & = & f_{1}\left(u_{1}\right) \\
\dot{u}_{2} & = & f_{2}\left(u_{1}, u_{2}\right) \\
\vdots & \vdots & \vdots \\
\dot{u}_{n} & = & f_{n}\left(u_{1}, \cdots, u_{n}\right)
\end{array}\right.
$$

where $f_{j}: \mathbb{R}^{j} \rightarrow \mathbb{R}, 1 \leq j \leq n$, are $C^{1}$ functions. Assume that there is $\xi>0$ such that $f_{j}\left(u_{1}, \cdots, u_{j}\right) u_{j}<0$ for all $\left(u_{1}, \cdots, u_{j}\right) \in \mathbb{R}^{j}$ such that $\left|u_{j}\right| \geq \xi$ and for all $1 \leq j \leq n$. Assume that all equilibria of (5.2) are hyperbolic. It follows from the results in [1] that the semigroup associated to (5.2) is gradient-like (therefore gradient). Now we consider a small non-autonomous perturbation of the above problem; that is,

$$
\left\{\begin{array}{ccc}
\dot{u}_{1} & = & f_{1}\left(u_{1}\right)+g_{1}\left(t, u_{1}, \cdots, u_{n}\right), \\
\dot{u}_{2} & = & f_{2}\left(u_{1}, u_{2}\right)+g_{2}\left(t, u_{1}, \cdots, u_{n}\right), \\
\vdots & \vdots & \vdots \\
\dot{u}_{n} & = & f_{n}\left(u_{1}, \cdots, u_{n}\right)+g_{n}\left(t, u_{1}, \cdots, u_{n}\right) .
\end{array}\right.
$$

If $g_{j}(t, \cdot): \mathbb{R}^{n} \rightarrow \mathbb{R}$ is a $C^{1}\left(\mathbb{R}^{n}, \mathbb{R}\right)$ function with the property that

$$
\sup _{t \in \mathbb{R}} \sup _{\left(u_{1}, \cdots, u_{n}\right) \in \mathbb{R}^{n}}\left\{\left|g_{j}\left(t, u_{1}, \cdots, u_{n}\right)\right|+\sum_{i=1}^{n}\left|\frac{\partial g_{j}}{\partial u_{i}}\left(t, u_{1}, \cdots, u_{n}\right)\right|\right\}
$$

is suitably small, it follows from the results in [5] that the evolution process $\left\{T_{g}(t, s)\right.$ $: t \geq s\}$ associated to (5.3) is gradient-like and, as a consequence of the results in Section 4, the pullback attractor of $\left\{T_{g}(t, s): t \geq s\right\}$ has a Morse-decomposition and a Lyapunov function.

One can easily see that cascade systems of semilinear parabolic problems can also be considered. 


\section{Conclusive Remarks}

In this paper we have presented a theory for the Morse-decomposition of pullback attractors of evolution processes suitable for the class of non-autonomous perturbations of semigroups. Our approach extends the notions and main results of the associated autonomous theory in [8, 20, 1. In particular, it is shown that a nonautonomous perturbation of a gradient semigroup is a gradient non-autonomous evolution process, in the sense that it has a (time-dependent) continuous (in the whole phase space) Lyapunov function. Moreover, this Lyapunov function behaves continuously with respect to the underlying perturbation.

Concerning the autonomous theory, two main remarks are well worth being emphasized:

a) According to Definition 1.5, a local attractor must be an isolated invariant family. This property allows us prove that our definition of local attractor extends the notion of local attractor of the autonomous case (see Theorem 1.6). Again, a hypothesis of uniform separation on the attractor-repeller pair (automatic in the autonomous case) ensures that the associated repeller is closed. In Remark 1.4 we discuss some properties of isolated invariant sets in the autonomous framework, among them, a dichotomous behavior of solutions around them (topological hyperbolicity).

b) In [1, restricted to the autonomous case, it is shown that gradient systems are stable under perturbation by showing that a gradient semigroup (with a Lyapunov functional) is gradient-like in the sense of Definition 2.5 (or Definition 2.8 in [1]) and that a gradient-like semigroup is gradient (has a Lyapunov function). Note that, in the non-autonomous framework, we do not have that the existence of a Lyapunov functional implies that the evolution process is gradient-like. This fact deserves further attention.

In a non-autonomous framework, Rasmussen 19 (see also Kloeden and Rasmussen [12]) adopts a different approach towards a Morse-decomposition for evolution processes, specially adapted for finite dimensional phase spaces. In particular, if $\{T(t, s): t \geqslant s\}$ is an invertible non-linear evolution process with pullback attractor $\{\mathcal{A}(t): t \in \mathbb{R}\}$, a compact invariant family $\{A(t): t \in \mathbb{R}\}$ with $A(t) \subseteq \mathcal{A}(t)$ for every $t \in \mathbb{R}$ is defined as a local pullback attractor if there exists $\eta>0$ such that

$$
\lim _{s \rightarrow-\infty} \operatorname{dist}_{H}\left(T(t, s) \mathcal{O}_{\eta}(A(s)), A(t)\right)=0, \text { for all } t \in \mathbb{R},
$$

where $\mathcal{O}_{\eta}(A(s)) \doteq\left\{x \in \mathcal{A}(s): \operatorname{dist}_{H}(x, A(s))<\eta\right\}$, for all $s \in \mathbb{R}$.

Observe that a local pullback attractor is a pullback attractor with respect to the attraction universe $\mathcal{D}$ containing all the families $\left\{\mathcal{O}_{\zeta}(A(t)): t \in \mathbb{R}\right\}$ where $\zeta \in(0, \eta]$. On the other hand, a compact invariant family $A^{*}=\left\{A^{*}(t): t \in \mathbb{R}\right\}$ with $A^{*}(t) \subset \mathcal{A}(t)$ for every $t \in \mathbb{R}$ is called a local repeller if there exists $\eta>0$ such that

$$
\lim _{s \rightarrow-\infty} \operatorname{dist}_{H}\left(T(s, t) \mathcal{O}_{\eta}\left(A^{*}(t)\right), R(s)\right)=0, \text { for all } t \in \mathbb{R} .
$$

This is not clear, and we think that it would need deeper research if similar results to ours in this paper could be developed from this approach of pullback attraction for the local attractors. The same kind of situation is found in the case of random dynamical systems (see Liu [14]) related to some stochastic differential equations. 


\section{ACKNOWLEDGEMENT}

The authors wish to express their gratitude to the anonymous referees whose comments and suggestions have greatly improved the readability of the article.

\section{REFERENCES}

[1] E. R. Aragão-Costa, T. Caraballo, A. N. Carvalho and J.A. Langa, Stability of gradient semigroups under perturbation, Nonlinearity 24 (2011), 2099-2117. MR2805595

[2] E. R. Aragão-Costa, T. Caraballo, A. N. Carvalho and J.A. Langa, Continuity of Lyapunov functions and energy level for a generalized gradient system, Topological Methods in Nonlinear Analysis 39 (2012), 57-82. MR2934334

[3] A. V. Babin and M. I. Vishik, Attractors in Evolutionary Equations, Studies in Mathematics and its Applications 25, North-Holland Publishing Co., Amsterdam (1992). MR 1156492 (93d:58090)

[4] T. Caraballo, A. N. Carvalho, J. A. Langa and L. F. Rivero, A gradient-like non-autonomous evolution processes, International Journal of Bifurcation and Chaos 20 (9) (2010), 2751-2760. MR 2738731 (2011i:37108)

[5] A. N. Carvalho and J. A. Langa, An extension of the concept of gradient processs which is stable under perturbation, J. Differential Equations 246 (2009), 2646-2668. MR 2503016 (2010a:37159)

[6] A. N. Carvalho, J. A. Langa, J. C. Robinson, On the continuity of pullback attractors for evolution processes, Nonlinear Analysis 71 (2009), 1812-1824. MR2524394(2010f:37140)

[7] A. N. Carvalho, J. A. Langa, J. C. Robinson, A. Suárez, Characterization of non-autonomous attractors of a perturbed gradientsystem, J. Differential Equations 236 (2007) 570-603. MR 2322025 (2008e:37075)

[8] C. Conley, Isolated invariant sets and the Morse index. CBMS Regional Conference Series in Mathematics, 38, American Mathematical Society, Providence, R.I. (1978). MR511133 (80c:58009)

[9] J. K. Hale Asymptotic Behavior of Dissipative Systems, Mathematical Surveys and Monographs Number 25 (American Mathematical Society, Providence, RI) (1988). MR941371 (89g:58059)

[10] D. Henry, Geometric Theory of Semilinear Parabolic Equations, Lecture Notes in Mathematics 840, Springer-Verlag, Berlin (1981). MR610244 (83j:35084)

[11] M. Hurley, Chain recurrence, semiflows and gradients, J. Dyn. Diff. Equations 7 (1995), 437-456. MR1348735 (96k:58190)

[12] P.E. Kloeden, M. Rasmussen, Nonautonomous Dynamical Sytems, Mathematical Surveys and Monographs, AMS 2011. MR 2808288

[13] O. A. Ladyzhenskaya, Attractors for semigropus and Evolution Equations, Cambridge University Press, Cambridge (1991). MR1133627 (92k:58040)

[14] Z. Liu, The random case of Conley's theorem: III. Random semiflow case and Morse decomposition, Nonlinearity 20 (2007), 2773-2791. MR2368325 (2008m:37088)

[15] K. Mischaikow, H. Smith and H. R. Thieme, Asymptotically autonomous semiflows: Chain recurrent and Lyapunov functions, Trans. Amer. Math. Soc. 347 (5) 1669-1685 (1995). MR 1290727 (95h:34063)

[16] D. E. Norton, The fundamental theorem of dynamical systems, Comment. Math., Univ. Carolinae 36 (3) (1995), 585-597. MR.1364499(97a:58110)

[17] M. Patrão, Morse decomposition of semiflows on topological spaces, J. Dyn. Diff. Equations 19 (1) (2007), 181-198. MR2279951 (2007m:37033)

[18] M. Patrão and Luiz A.B. San Martin, Semiflows on topological spaces: Chain transitivity and semigroups, J. Dyn. Diff. Equations 19 (1) (2007), 155-180. MR2279950(2007m:37032)

[19] M. Rasmussen, Morse decomopsitions of nonautonomous dynamical systems, Trans. Amer. Math. Soc. 359 (10) (2007), 5091-5115. MR 2320661 (2009a:34086)

[20] K. P. Rybakowski, The homotopy index and partial differential equations, Universitext, Springer-Verlag (1987). MR910097 (89d:58025)

[21] R. Temam, Infinite-Dimensional Dynamical Systems in Mechanics and Physics, SpringerVerlag, Berlin (1988; second edition 1996). MR:953967 (89m:58056) 
Instituto de Ciências Matemáticas e de ComputaÇão, Universidade de São PauloCampus de São Carlos, Caixa Postal 668, 13560-970 São Carlos SP, Brazil

E-mail address: ritis@icmc.usp.br

Departamento de Ecuaciones Diferenciales y Análisis Numérico, Universidad de Sevilla, Apdo. de Correos 1160, 41080-Sevilla Spain

E-mail address: caraball@us.es

Instituto de Ciências Matemáticas e de ComputaÇão, Universidade de São PauloCampus de São Carlos, Caixa Postal 668, 13560-970 São Carlos SP, Brazil

E-mail address: andcarva@icmc.usp.br

Departamento de Ecuaciones Diferenciales y AnÁlisis Numérico, Universidad de Sevilla, Apdo. de Correos 1160, 41080-Sevilla Spain

E-mail address: langa@us.es 